\title{
Global embeddings for branes at toric singularities
}

\author{
Vijay Balasubramanian, ${ }^{a}$ Per Berglund, ${ }^{b}$ Volker Braun, ${ }^{c}$ and Iñaki García-Etxebarria ${ }^{d}$ \\ ${ }^{a}$ Department of Physics and Astronomy, University of Pennsylvania, \\ Philadelphia, PA 19104-6396, U.S.A. \\ ${ }^{b}$ Department of Physics, University of New Hampshire, \\ Durham, NH 03824, U.S.A. \\ ${ }^{c}$ Dublin Institute for Advanced Studies, \\ Dublin, Ireland \\ ${ }^{d}$ Theory Group, Physics Department, CERN, \\ CH-1211, Geneva 23, Switzerland \\ E-mail: vijay@physics.upenn.edu, per.berglund@unh.edu, vbraun@dias.ie, \\ inaki@mail.cern.ch
}

ABSTRACT: We describe how local toric singularities, including the Toric Lego construction, can be embedded in compact Calabi-Yau manifolds. We study in detail the addition of D-branes, including non-compact flavor branes as typically used in semi-realistic model building. The global geometry provides constraints on allowable local models. As an illustration of our discussion we focus on $D 3$ and $D 7$-branes on (the partially resolved) $\left(d P_{0}\right)^{3}$ singularity, its embedding in a specific Calabi-Yau manifold as a hypersurface in a toric variety, the related type IIB orientifold compactification, as well as the corresponding F-theory uplift. Our techniques generalize naturally to complete intersections, and to a large class of F-theory backgrounds with singularities.

KEYwords: Compactification and String Models, D-branes, F-Theory, Superstring Vacua ARXIV EPRINT: 1201.5379 


\section{Contents}

1 Introduction 2

2 Global embedding of toric singularities 4

2.1 Generalities 5

$2.2 d P_{0} \rightarrow M_{d P_{0}}$

2.3 The hyperconifold $Y^{3,0} \rightarrow M_{Y^{3,0}} \quad 9$

$2.4\left(d P_{0}\right)^{3} \rightarrow M_{\left(d P_{0}\right)^{3}} \quad 13$

3 Adding D-branes to the model $\quad 17$

$\begin{array}{ll}3.1 \text { Blowing up the fractional branes } & 19\end{array}$

3.1.1 The $\Psi$ map 19

3.1.2 Computing the spectrum 24

3.2 Flavor $D 7$ branes $\quad 25$

$\begin{array}{lll}3.3 & D 5 \text {-brane tadpole } & 27\end{array}$

$4 \quad$ Type IIB orientifolds $\quad 29$

$\begin{array}{lll}4.1 & \text { Generalities } & 29\end{array}$

4.2 Sign orientifolds: $M_{d P_{0}} \quad 30$

4.3 Permutation orientifolds: $M_{\left(d P_{0}\right)^{3}} \quad 31$

5 Hybrid embeddings $\quad 34$

$\begin{array}{lll}5.1 & M_{\left(d P_{0}\right)^{3}} & 34\end{array}$

6 Mapping the landscape of singularities $\quad 37$

6.1 How singular is the KS landscape? 37

6.2 Singularities vs. Hodge numbers 38

$\begin{array}{ll}\text { 6.3 The most singular polytope } & 39\end{array}$

6.4 Toric del Pezzo singularities $\quad 40$

$6.5 Y^{(p, q)}$ and $L^{(a, b, a)}$ cones 40

6.6 Toric Lego models 41

6.7 Computer implementation 42

7 Conclusions and generalizations $\quad 42$

7.1 More general F-theory bases 43

7.2 Complete intersections and toroidal orbifolds 43 


\section{Introduction}

A convenient bottom-up approach to model building in string theory is to consider Dbranes placed at local geometric singularities in a compactification manifold [1-6]. The local properties of the singularities, together with the D-branes, determine the matter and gauge content of the theory. In a previous paper [7] (see also [8, 9]) we described a construction, called Toric Lego, in which local toric singularities supporting different desirable sectors of a low-energy field theory (e.g. a standard model sector, a supersymmetry breaking sector, and a dark matter sector) can be glued together geometrically to construct a larger parent singularity, resulting in a consistent, anomaly free, field theory encompassing the different sectors. The sizes of collapsing cycles connecting the different daughter singularities fix the masses of messengers communicating between the different low-energy field theory sectors. This sort of modular model building is especially convenient because it makes it possible to separately engineer field theories realizing specific useful properties, which can then be glued together to form a complete model.

Any local construction of this kind faces a basic question - can the desired local singularity be embedded consistently in a globally well-defined compact Calabi-Yau manifold? In particular, global tadpole cancellation conditions in general require the introduction of orientifold planes with negative D-brane charges, which may be incompatible with the local structure and embedding we have chosen, as we will see in some later examples.

In this paper we make progress towards an algorithm for systematically producing tadpole-satisfying global embeddings of toric singularities in type IIB compactifications, e.g. those in the Toric Lego models [7]. The natural place to look for such global realizations is in terms of Calabi-Yau manifolds given as hypersurfaces in toric varieties. This task is made easier thanks to Kreuzer and Skarke's classification of toric varieties in terms of reflexive polyhedra in four dimensions [10]. The class of global models that we study can all be described as follows: consider a Calabi-Yau manifold, $M$, described in terms of a hypersurface constraint in a four dimensional toric variety $\mathcal{A}_{\nabla}$ obtained from the four dimensional polytope $\nabla$. The global embedding of the local toric singularity is then obtained by determining whether one of the three dimensional cones obtained in a given (fine) triangulation of $\nabla$ is the cone over the two dimensional toric diagram of the local singularity.

Failure to find a global realization through our procedure does not imply that the local singularity can have no globally well-defined embedding - it simply means that there is no embedding within the class of models considered. For example, there may be an embedding into a Calabi-Yau constructed as a complete intersection within a higher dimensional toric variety. This class of embeddings can also be studied using a natural extension of our methods, see the discussion in section 7.2.

As mentioned above, the introduction of D-branes in the global realization of the toric singularities means that we naturally have to include orientifold planes as well. Since we are mainly interested in gauge theories with $\mathrm{U}(N)$ factors only, we focus on $\mathbb{Z}_{2}$ permutation involutions in which pairs of branes at singularities are exchanged. These type IIB compactifications are then up-lifted to (singular) Calabi-Yau fourfolds in F-theory. One can in 
fact avoid the step of constructing a IIB orientifold background, and directly construct an F-theory compactification. We comment further on this possibility in section 7.1.

Naturally, there are other important constraints that a realistic global model must satisfy beyond tadpole cancellation, such as moduli stabilization at the desired values for the local model, and realistic supersymmetry breaking at a local minimum of the potential. In this paper we content ourselves with providing a way to find large classes of embeddings which satisfy $D 7$ tadpole cancellation, with the expectation that in the class of global models that we find, some models also have working moduli stabilization and supersymmetry breaking. We plan to return to this important question in future work.

\section{Relation to previous work}

There are various approaches to the problem of bottom-up model building in the literature. In the following we quickly review the main similarities and differences of the existing constructions to our approach.

A recent class of F-theory models [11-15] embeds a (typically SU(5)) GUT brane wrapping a small but not collapsed cycle into F-theory. The gauge dynamics comes from the SU(5) stack, and the breaking into the standard model comes from fluxes living on the brane. In the quiver models we study, on the other hand, the branes wrap zero size cycles, and the breaking into factors happens due to $\alpha^{\prime}$ corrections, which modify the stability conditions of the branes from those at large volume. So an essential distinction is that the models in [11-15] live in a regime where $\alpha^{\prime}$ corrections can be ignored, while these $\alpha^{\prime}$ corrections are essential for us. A related important difference with the models that we analyze in this paper is that generally one does not want the orientifold to intersect the quiver locus, while this is unavoidable (and desirable) in realistic F-theory GUT models.

Previous works $[16,17]$ deal with the IIB/F-theory embedding of $d P_{k}$ singularities with $k>3$, which are non-toric. In addition to the toric vs. non-toric distinction, a more important difference with these studies is whether one looks for the singular locus in Kähler or complex structure moduli space. We consider singularities in the Kähler moduli space of the ambient space, which are then inherited by the Calabi-Yau hypersurface. It is certainly possible, and necessary in the case of non-toric singularities, to obtain the singularity from a degeneration of the Calabi-Yau hypersurface, appearing at particular loci in the complex structure moduli space of the Calabi-Yau. One advantage of our choice is that we gain a way of formulating the search in purely combinatorial terms. Thus, the analysis can be performed on a computer, and allows us to find a plethora of possible embeddings.

Another difference with previous studies is that we discuss in detail flavor D7 branes. This introduces a number of complications, which we analyze in section 3 , but it is necessary if one wants to embed many of the semi-realistic models in the literature (see [18] for the state of the art).

Branes at toroidal orbifolds (see [19] for a review with further references), while superficially very different from the models that we consider in this paper, can in fact often be incorporated into our framework, as we discuss further in section 7.2. Along the same lines, the topic of landscape scans for realistic physics has been explored in detail in the context of intersecting branes on toroidal orbifolds [20-25]. These papers focus on the 
open string sector, while we focus on the closed string sector. Doing a combined search would definitely be desirable, and ties together nicely with the question of general F-theory embeddings discussed in section 7.1.

\section{Embedding procedure and layout of this paper}

The embedding prescription we propose proceeds in four steps:

1. One first finds an embedding of the singularity into a compact Calabi-Yau (or Ftheory base, in which case one can skip step 3 below). Rather than searching for the singular point in the Calabi-Yau itself, we search for a curve of singularities in an ambient toric space. By dimensional counting the Calabi-Yau hypersurface intersects the curve of singularities at a point. We describe how to do this in some particular examples in section 2 .

In addition to just looking for particular models, we can use our method to get an idea of how generic it is to find semi-realistic singular loci in the landscape of CalabiYau spaces. We do such a scan in section 6, focusing on the list of models produced by Kreuzer and Skarke [10].

2. Once we have the geometry, we introduce branes. The discussion of the local model at the quiver locus is most easily given in terms of quiver representations, while global considerations such as tadpole cancellation are most easily studied in terms of the algebraic geometry of sheaves. We thus need a dictionary between both languages. We review the known results for the gauge nodes in section 3, and extend them to also cover flavor branes.

3. Tadpole cancellation forces us to introduce orientifolds. In section 4 we review how orientifolds can be introduced into our class of constructions, and how to construct the resulting quotient of the Calabi-Yau.

4. The quotient constructed in the previous step can be used as a basis for a F-theory compactification. One advantage of lifting to F-theory is that this automatically takes care of the 7-brane tadpoles. Formulating the discussion in this way also allows for easy generalization to compactifications in which the quiver sector is only locally weakly coupled. An important constraint is that the discriminant reproduces the local flavor structure close to the quiver theory. We illustrate how to satisfy this constraint in a particular example in section 5 .

We summarize our results in section 7 , together with some technically simple but physically interesting extensions of our work.

\section{Global embedding of toric singularities}

We now describe how a given local toric singularity is embedded in a compact Calabi-Yau manifold. This provides constraints on which local geometries can be realized in a global model. After a brief general discussion in section 2.1, we illustrate the method with a few 
examples. The first and simplest local geometry is $\mathbb{C}^{3} / \mathbb{Z}_{3}$, and is studied in section 2.2. In section 2.3 we consider the embedding of the hyperconifold $Y^{3,0}$, which can also be seen as two $\mathbb{C}^{3} / \mathbb{Z}_{3}$ singularities joined by a collapsed $\mathbb{P}^{1}$. After these warm-up examples, in section 2.4 , we analyze the main example in this paper, denoted $\left(d P_{0}\right)^{3}$. In the spirit of the Toric Lego construction [7], this geometry is obtained by joining three copies of $\mathbb{C}^{3} / \mathbb{Z}_{3}$.

We assume some rudimentary knowledge of the basics of toric geometry, good references are [26-28] and the excellent recent book [29]. The discussion in sections 2.2 to 2.4 is technical. We encourage those readers who are not interested in the details to proceed directly to the discussion in section 3, after a quick overview of our method in section 2.1.

\subsection{Generalities}

Toric Calabi-Yau varieties are necessarily non-compact [26]. Unfortunately, this makes a direct toric description of the compact Calabi-Yau embedding impossible. Nevertheless, Calabi-Yau spaces can be obtained as hypersurfaces (or, more generally, complete intersections) in ambient toric varieties (which are compact, and thus not Calabi-Yau) associated to reflexive ${ }^{1}$ polytopes. A famous example is the quintic Calabi-Yau threefold as a degree 5 hypersurface in the toric space $\mathbb{P}^{4}$.

In this paper we focus on Calabi-Yau spaces which are constructed in the above way. In order to make the discussion concrete, we restrict ourselves to the list of Calabi-Yau hypersurfaces constructed by Kreuzer and Skarke [10], although the discussion can be generalized with little effort to the case of complete intersections in toric spaces, see section 7.2.

In order to introduce singularities, we have two possibilities:

- Specialize the embedding equation to be singular. For example, take the quintic at the conifold point. The singular loci can, in principle, be read off from the PicardFuchs equations. But actually doing so is computationally difficult unless $h^{21}$ is very small. Moreover, identifying the position and type of singularities can be a difficult computational problem.

- Leave the embedding equation generic but make the ambient toric variety singular. The type of the singularities is then determined by the singularities in the ambient toric varieties, which can be read off by the comparatively simple combinatorics of the toric fan.

Since it is computationally much easier, we follow the second approach in this paper and construct curves of singularities in the ambient toric variety that then intersect the CalabiYau hypersurface in points. Our general search strategy follows from the following simple observation: consider a reflexive polytope $\nabla$ describing the ambient toric space $\mathcal{A}_{\nabla}$. Let us denote by $\mathcal{F}_{2}$ the toric diagram describing our local Calabi-Yau singularity $X{ }^{2}$ Now assume that $\nabla$ has $\mathcal{F}_{2}$ as one of its two-dimensional faces, i.e., the toric fan for $X$ is included

\footnotetext{
${ }^{1}$ In technical terms, we consider crepant partial resolutions of Fano toric varieties. This condition ensures that a generic Calabi-Yau threefold hypersurface is a smooth manifold.

${ }^{2}$ We remind the reader that every toric, non-compact, Calabi-Yau threefold can be completely specified by a two-dimensional convex diagram, called the toric diagram. We will see explicit examples below.
} 
as a $3 \mathrm{~d}$ cone in $\nabla$. This implies that there is a patch of $\mathcal{A}_{\nabla}$ that looks like $X \times \mathbb{C}^{*}$. Now, if we consider a Calabi-Yau hypersurface, due to simple dimension counting we expect the hypersurface to intersect the curve of singularities at a copy of $X$. Once we find a candidate ambient space $\mathcal{A}_{\nabla}$ we can compute the topology of the divisors in $\mathcal{F}_{2}$, and make sure that they agree with what one expects from the local structure of $X$. Since we have a toric description of the whole setup, this can be done straightforwardly and we show this explicitly in some of the examples below. The search strategy is then rather obvious, and easily implemented on a computer: we go through the 473,800,776 [10] reflexive polytopes in $4 \mathrm{~d}$, and compare each of the $2 \mathrm{~d}$ faces of each polytope with the toric diagram of the singularity that we wish to embed.

A similar argument would apply if we search for two-dimensional faces containing the toric diagram for the singularity, in such a way that the local geometry can be partially resolved to the geometry of interest. The $\left(d P_{0}\right)^{n}$ examples with $n>1$ that we study below provide for an illustration of this idea: upon partial resolution they give rise to local $\mathbb{C}^{3} / \mathbb{Z}_{3}$ singularities. Although we will not focus much on this possibility in this paper, it is a consistent search strategy, and leads to interesting models.

As a technical remark, let us emphasize that since we are interested in singular spaces the cones in our fan are not necessarily simplicial. It is nevertheless technically easier to work with spaces with at most orbifold singularities. Thus, in our examples we perform a partial resolution, and then make sure that the blown-up cycles can be contracted to zero size by moving in Kähler moduli space without making the volume of the whole Calabi-Yau space vanish.

Once we have the local geometry embedded in a compact Calabi-Yau manifold we can turn to the open string sector and the inclusion of D-branes. Before we do so in sections 3 and onwards, however, we illustrate the embedding procedure in some simple examples. These examples are obtained by performing the scan over reflexive polytopes mentioned above. In order to make the discussion as clear as possible we have hand-picked some particularly simple reflexive polytopes having the singularities that we want to analyze. We leave the exhaustive scan over $4 \mathrm{~d}$ reflexive polytopes to section 6 .

\section{$2.2 d P_{0} \rightarrow M_{d P_{0}}$}

We start by considering the embedding of a particularly simple example of a local geometry, namely $\mathbb{C}^{3} / \mathbb{Z}_{3}$ with the $\mathbb{Z}_{3}$ orbifold action on the $\mathbb{C}^{3}$ coordinates $(x, y, z)$ given by

$$
(x, y, z) \rightarrow(\omega x, \omega y, \omega z), \quad \omega=e^{2 \pi i / 3} .
$$

The $\mathbb{C}^{3} / \mathbb{Z}_{3}$ singularity can be described as the singular limit of the local $\mathbb{P}^{2}$ Calabi-Yau geometry, that is, the total space of the $\mathcal{O}_{\mathbb{P}^{2}}(-3)$ bundle. $\mathbb{P}^{2}$ is a del Pezzo surface, known in this context as $d P_{0}$, hence our notation. ${ }^{3}$ When the $d P_{0}$ surface is contracted to zero size we obtain the $\mathbb{C}^{3} / \mathbb{Z}_{3}$ orbifold. We embed this local singularity into an elliptic fibration over $\mathbb{P}^{2}$, which we call $M_{d P_{0}}$.

\footnotetext{
${ }^{3}$ Note that $d P_{0}$ is also referred to as a del Pezzo surface of degree 9 . We follow the physics tradition and denote by $d P_{k}$ a del Pezzo surface of degree $d=9-k$.
} 


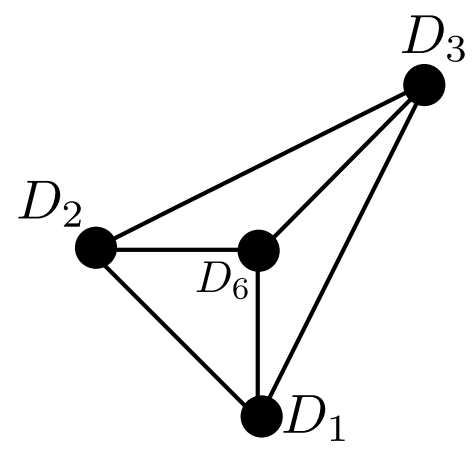

Figure 1. The polytope for $d P_{0}$, or equivalently, the toric diagram for the (resolved) $\mathbb{C}^{3} / \mathbb{Z}_{3}$. The particular assignment of labels to the vertices follows from the ones in the global embedding.

The three-dimensional toric variety $\mathbb{C}^{3} / \mathbb{Z}_{3}$ has a very simple description in terms of a toric diagram, see figure 1 . By lifting the toric diagram into its corresponding $3 \mathrm{~d}$ cone by adding an extra coordinate, we have that the coordinates of the vertices in a particular coordinate system are given by $v_{1}=(0,-1,1), v_{2}=(-1,0,1)$ and $v_{3}=(1,1,1)$. The interior point of the diagram is also important, and we denote it by $v_{6}=(0,0,1)$. We can associate a (complex) coordinate $x_{i}$ to each $v_{i}$, where $x_{i}=0$ gives the divisor $D_{i}$. Hence, to each vertex in the toric diagram we associate a 4-cycle in the non-compact Calabi-Yau manifold, and the 2-cycles are given by the lines connecting the vertices. In the singular $\mathbb{C}^{3} / \mathbb{Z}_{3}$ case we remove the $v_{6}$ ray, and the singular point in $\mathbb{C}^{3} / \mathbb{Z}_{3}$ is represented by the interior face of the polytope. These coordinates of the vertices $v_{i}$ satisfy the linear relation $v_{1}+v_{2}+v_{3}-3 v_{6}=0$. Turning the coefficients of the relation into charges under a $\mathbb{C}^{*}$, we thus obtain a gauged linear $\Sigma$ model given by the gauge symmetry

$$
\left(x_{1}, x_{2}, x_{3}, x_{6}\right) \rightarrow\left(\lambda x_{1}, \lambda x_{2}, \lambda x_{3}, \lambda^{-3} x_{6}\right) .
$$

We embed this local geometry in the Calabi-Yau hypersurface $M_{d P_{0}}$ in the resolved weighted projective space $\mathbb{P}_{[1: 1: 1: 6: 9]}^{4}$. This Calabi-Yau threefold is also known as the toric elliptic fibration over $\mathbb{P}^{2}$ with Hodge numbers $\left(h^{11}, h^{21}\right)=(2,272)$ [30-32]. To understand how the local geometry of the resolved $\mathbb{C}^{3} / \mathbb{Z}_{3}$ singularity is embedded in $M_{d P_{0}}$, we describe the latter as a hypersurface in a four dimensional toric variety $\mathcal{A}_{d P_{0}}$. The ambient space $\mathcal{A}_{d P_{0}}$ has an associated reflexive polytope with coordinates

\begin{tabular}{rrrrrr}
$x_{1}$ & $x_{2}$ & $x_{3}$ & $x_{4}$ & $x_{5}$ & $x_{6}$ \\
\hline 0 & -1 & 1 & 0 & 0 & 0 \\
-1 & 0 & 1 & 0 & 0 & 0 \\
2 & 2 & 2 & -1 & 0 & 2 \\
3 & 3 & 3 & 0 & -1 & 3
\end{tabular}

Notice how the polytope has a face in the $(\bullet, \bullet, 2,3)$ plane given by the diagram in figure 1 , which we recognize as the toric diagram of $d P_{0}$. This is thus a candidate embedding of our desired local singularity, as we verify in more detail momentarily.

In order to establish that the above Calabi-Yau hypersurface is indeed the resolution of a compact Calabi-Yau variety with a $\mathbb{C}^{3} / \mathbb{Z}_{3}$ singularity, a few more details have to be 


\begin{tabular}{|c|cccccc|}
\hline$\ell \cdot D$ & $x_{1}$ & $x_{2}$ & $x_{3}$ & $x_{4}$ & $x_{5}$ & $x_{6}$ \\
\hline$\ell_{1}$ & 1 & 1 & 1 & 0 & 0 & -3 \\
$\ell_{2}$ & 0 & 0 & 0 & 2 & 3 & 1 \\
\hline
\end{tabular}

Table 1. Mori cone for the ambient space in which $M_{d P_{0}}$, an elliptic fibration over $\mathbb{P}^{2}$, is defined as a hypersurface. Note that the Mori cones for the ambient toric variety, $\mathcal{A}_{d P_{0}}$, and the Calabi-Yau manifold, $M_{d P_{0}}$, are identical in this case, unlike the examples in sections 2.3 and 2.4 .

checked. Since the ambient space has to account for the local singularity of interest, there exist in general many Calabi-Yau phases realizing the local geometry, depending on how the singularity is resolved [33, 34]. Given a particular resolution of the ambient singularity, corresponding to a fine triangulation of the associated polytope, we compute the Mori cone, that is, the cone in $H_{2}\left(\mathcal{A}_{d P_{0}}, \mathbb{Z}\right)=H_{2}\left(M_{d P_{0}}, \mathbb{Z}\right)=\mathbb{Z}^{2}$ spanned by holomorphic curves. For $\mathcal{A}_{d P_{0}}$ there is a unique fine triangulation, ${ }^{4}$ resulting in the Mori cone given in table 1 . The fan of the ambient toric variety for this unique triangulation is spanned by the 9 generating cones

$$
\begin{aligned}
\mathcal{F}\left(\mathcal{A}_{d P_{0}}\right)= & \left\langle\left\langle x_{1} x_{2} x_{4} x_{5}\right\rangle,\left\langle x_{1} x_{2} x_{4} x_{6}\right\rangle,\left\langle x_{1} x_{2} x_{5} x_{6}\right\rangle,\left\langle x_{1} x_{3} x_{4} x_{5}\right\rangle,\left\langle x_{1} x_{3} x_{4} x_{6}\right\rangle,\right. \\
& \left.\left\langle x_{1} x_{3} x_{5} x_{6}\right\rangle,\left\langle x_{2} x_{3} x_{4} x_{5}\right\rangle,\left\langle x_{2} x_{3} x_{4} x_{6}\right\rangle,\left\langle x_{2} x_{3} x_{5} x_{6}\right\rangle\right\rangle .
\end{aligned}
$$

The Stanley-Reisner ideal for this triangulation is

$$
S R\left(\mathcal{A}_{d P_{0}}\right)=\left\langle x_{1} x_{2} x_{3}, x_{4} x_{5} x_{6}\right\rangle
$$

The Kähler cone of $M_{d P_{0}}$, which is the dual of the Mori cone for $M_{d P_{0}}$, agrees with the Kähler cone of the ambient space $\mathcal{A}_{d P_{0}}$

$$
\begin{aligned}
\mathcal{K}\left(\mathcal{A}_{d P_{0}}\right)=\mathcal{K}\left(M_{d P_{0}}\right) & =\left\{D \in \operatorname{Cl}\left(M_{d P_{0}}\right) \mid D \cdot \ell_{i}>0, i=1,2\right\} \\
& =\operatorname{span}\left\{V\left(x_{1}\right), V\left(x_{6}\right)+3 V\left(X_{1}\right)\right\} .
\end{aligned}
$$

As we will see in sections 2.3 and 2.4 it is in general not the case that the Kähler cones (and hence the Mori cones) agree between the ambient space and the Calabi-Yau hypersurface, but for this simple example they do.

We can use this information to write down the hypersurface equation of the Calabi-Yau manifold. Since the entries for each of the Mori generators also gives the scaling behavior of the $x_{i}$ homogeneous coordinate under the corresponding $\mathbb{C}^{*}$ in table 1 , we find that

$$
x_{5}^{2}=x_{4}^{3}+x_{4} x_{6}^{4} f_{12}\left(x_{1}, x_{2}, x_{3}\right)+x_{6}^{6} g_{18}\left(x_{1}, x_{2}, x_{3}\right)
$$

This is indeed the Weierstrass form of an elliptic fibration over $\mathbb{P}^{2}$. Note that the generic toric fiber of the ambient space $\mathcal{A}_{d P_{0}}$ is the weighted projective plane $\mathbb{P}_{[3: 2: 1]}^{2}$. It is a

\footnotetext{
${ }^{4}$ In general the global geometry have multiple fine triangulations, each of which gives rise to a different Calabi-Yau manifold, once the hypersurface condition has been imposed. As we will see in sections 2.3 and 2.4, it is not always the case that all Calabi-Yau phases preserve the local structure of the partially resolved singularity one would like to embed.
} 
general fact for such toric fibrations that the Calabi-Yau hypersurface is automatically in Weierstrass form, which is often used for computational simplicity. This particular CalabiYau hypersurface has also been used for Large Volume Scenario models in type IIB flux compactifications [35].

We now parametrize the Kähler form $\omega(t)$ by $t_{1}$ and $t_{2} \geq 0$, that is,

$$
\omega=t_{1} V\left(x_{1}\right)+t_{2}\left(3 V\left(x_{1}\right)+V\left(x_{6}\right)\right) .
$$

In these coordinates, the volume of $M_{d P_{0}}$ is

$$
\int_{M_{d P_{0}}} \omega^{3}=3 t_{1}^{2} t_{2}+9 t_{1} t_{2}^{2}+9 t_{2}^{3} .
$$

The volumes $\operatorname{Vol}\left(D_{i}\right)=\frac{1}{3} \partial_{t_{i}} \operatorname{Vol}\left(M_{d P_{0}}\right)$ of the dual 4-cycles are

$$
\begin{aligned}
& \tau_{1}=2 t_{1} t_{2}+3 t_{2}^{2} \\
& \tau_{2}=t_{1}^{2}+6 t_{1} t_{2}+9 t_{2}^{2} .
\end{aligned}
$$

In order to see the local geometry, we would like to identify the contracting $d P_{0}$. Clearly, this is the divisor $D_{6} \subset M_{d P_{0}}$ associated to the additional ray required to refine the face fan (the most coarse triangulation) to the smooth resolution. That this is indeed a $d P_{0}$ surface is illustrated by the Chern numbers

$$
\int_{D_{6}} c_{1}^{2}\left(T D_{6}\right)=9, \quad \int_{D_{6}} c_{2}\left(T D_{6}\right)=3
$$

Its volume is given by

$$
\int_{D_{6}} \omega^{2}=t_{1}^{2}
$$

so we can indeed contract $D_{6}$ to zero volume by sending $t_{1} \rightarrow 0$ while the Calabi-Yau volume stays finite as long as we keep $t_{2}$ finite.

\subsection{The hyperconifold $Y^{3,0} \rightarrow M_{Y^{3,0}}$}

The previous example serves as a good introduction to our methods, but it is too simple - it is a well-known fact that one can contract a del Pezzo surface in a Calabi-Yau to zero size. In this section we present an example with a non-del Pezzo singularity. This geometry serves as a simple illustration of the techniques in a slightly more involved case than the one studied in the previous section. However, this example is not particularly useful as a perturbative IIB orientifold background since it does not have a $\mathbb{Z}_{2}$ involution acting on the ambient polytope. Hence, we will not try to put an open string sector on it in coming sections.

Let us consider a local model with two sectors, each of which is locally $\mathbb{C}^{3} / \mathbb{Z}_{3}$. This local geometry, described by the toric diagram in figure 2 , is the $\mathbb{Z}_{3}$ orbifold of the conifold, also known as the $Y^{3,0}$ singularity. A possible global embedding of the local geometry is 


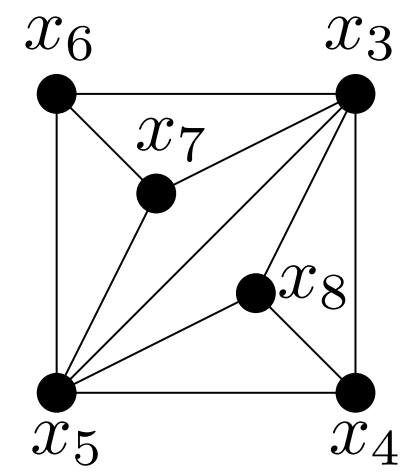

Figure 2. $Y^{3,0}$ resolved into two $d P_{0}$ 's separated by a $\mathbb{P}^{1}$. We have skewed the perspective slightly to ease visualization. The actual position of the points in the two-dimensional plane can be obtained by forgetting the last two coordinates in the polytope in eq. (2.13).

given by the reflexive polytope with vertices

\begin{tabular}{rrrrrrrrr}
$x_{1}$ & $x_{2}$ & $x_{3}$ & $x_{4}$ & $x_{5}$ & $x_{6}$ & $x_{7}$ & $x_{8}$ & $x_{9}$ \\
\hline 0 & 0 & 1 & 0 & -1 & 0 & 0 & 0 & 0 \\
0 & 0 & 1 & -1 & 0 & 2 & 1 & 0 & 1 \\
-1 & 0 & 2 & 2 & 2 & 2 & 2 & 2 & 1 \\
0 & -1 & 3 & 3 & 3 & 3 & 3 & 3 & 1
\end{tabular}

Notice that in order to resolve a $\mathbb{Z}_{2}$ singularity appearing when compactifying the local singularity, we have to include an extra point $\left(x_{9}\right)$ along the edge given by $x_{2}$ and $x_{6}$, in addition to the two points that were added to the polytope that was used to describe $M_{d P_{0}}$. The resulting Calabi-Yau manifold, $M_{Y^{3,0}}$, has Hodge numbers $\left(h_{11}, h_{21}\right)=(5,215)$. The number of Kähler deformations can be accounted for in following way. One Kähler modulus measures the volume of the curve resolving $Y^{3,0} \rightarrow \mathbb{C}^{3} / \mathbb{Z}_{3} \times \mathbb{C}^{3} / \mathbb{Z}_{3}$. There are another two Kähler moduli measuring the volumes of the two exceptional $\mathbb{P}^{2} \mathrm{~s}$. In the global embedding, the elliptic fiber (which may or may not be realized in a given phase) contributes one additional Kähler modulus. Finally, the above mentioned divisor, $D_{9}$, associated to the point $x_{9}$, gives the last modulus in the Calabi-Yau manifold.

We find seven different fine star triangulations of this polytope, two of which give rise to an elliptic fibration. Four of the remaining five triangulations reproduce the triangulation of the local geometry given in figure 2. We focus on one of the latter four triangulations. In this case, unlike the aforementioned elliptic fibration $M_{d P_{0}} \rightarrow \mathbb{P}^{2}$, the actual Mori cone of the Calabi-Yau hypersurface $M_{Y^{(3,0)}}$ is strictly smaller than the Mori cone of the ambient space $\mathcal{A}_{Y^{(3,0)}}$, because some of the curves in $\mathcal{A}_{Y^{(3,0)}}$ do not lie in $M_{Y^{(3,0)}}$.

Let us show this in detail. The chosen triangulation gives rise to a fan spanned by the following 19 cones

$$
\begin{aligned}
\mathcal{F}\left(\mathcal{A}_{Y^{(3,0)}}\right)= & \left\langle x_{1} x_{2} x_{3} x_{4}\right\rangle,\left\langle x_{1} x_{2} x_{3} x_{9}\right\rangle,\left\langle x_{1} x_{2} x_{4} x_{5}\right\rangle,\left\langle x_{1} x_{2} x_{5} x_{9}\right\rangle,\left\langle x_{1} x_{3} x_{4} x_{8}\right\rangle, \\
& \left\langle x_{1} x_{3} x_{5} x_{7}\right\rangle,\left\langle x_{1} x_{3} x_{5} x_{8}\right\rangle,\left\langle x_{1} x_{3} x_{6} x_{7}\right\rangle,\left\langle x_{1} x_{3} x_{6} x_{9}\right\rangle,\left\langle x_{1} x_{4} x_{5} x_{8}\right\rangle, \\
& \left\langle x_{1} x_{5} x_{6} x_{7}\right\rangle,\left\langle x_{1} x_{5} x_{6} x_{9}\right\rangle,\left\langle x_{2} x_{3} x_{4} x_{8}\right\rangle,\left\langle x_{2} x_{3} x_{5} x_{8}\right\rangle,\left\langle x_{2} x_{3} x_{5} x_{9}\right\rangle, \\
& \left.\left\langle x_{2} x_{4} x_{5} x_{8}\right\rangle,\left\langle x_{3} x_{5} x_{7} x_{9}\right\rangle,\left\langle x_{3} x_{6} x_{7} x_{9}\right\rangle,\left\langle x_{5} x_{6} x_{7} x_{9}\right\rangle\right\rangle
\end{aligned}
$$


and its Stanley-Reisner ideal is

$$
\begin{aligned}
S R\left(\mathcal{A}_{Y^{(3,0)}}\right)=\langle & x_{2} x_{6}, x_{2} x_{7}, x_{4} x_{6}, x_{4} x_{7}, x_{4} x_{9}, x_{8} x_{9}, x_{6} x_{8}, x_{7} x_{8}, x_{1} x_{2} x_{8}, \\
& \left.x_{3} x_{4} x_{5}, x_{1} x_{7} x_{9}, x_{3} x_{5} x_{6}, x_{1} x_{2} x_{3} x_{5}, x_{1} x_{3} x_{5} x_{9}\right\rangle
\end{aligned}
$$

reproducing the local geometry given in figure 2; for example, $x_{7}=x_{8}=0$ has no solution. In other words, the associated variety $\hat{D}_{7} \cap \hat{D}_{8}=\emptyset^{5}$ is empty instead of the expected codimension two. The Mori cone for $\mathcal{A}_{Y^{(3,0)}}$, given the above triangulation 2.14, is then given by

\begin{tabular}{c|rrrrrrrrrr}
$\hat{\ell} \cdot \hat{D}$ & $x_{1}$ & $x_{2}$ & $x_{3}$ & $x_{4}$ & $x_{5}$ & $x_{6}$ & $x_{7}$ & $x_{8}$ & $x_{9}$ & $K$ \\
\hline$\hat{\ell}_{1}$ & 1 & 0 & 0 & 0 & 0 & -2 & 1 & 0 & 3 & -3 \\
$\hat{\ell}_{2}$ & 0 & 0 & 1 & 1 & 1 & 0 & 0 & -3 & 0 & 0 \\
$\hat{\ell}_{3}$ & 0 & 0 & 1 & 0 & 1 & 1 & -3 & 0 & 0 & 0 \\
$\hat{\ell}_{4}$ & 0 & 1 & -1 & 0 & -1 & 0 & 3 & 0 & -2 & 0 \\
$\hat{\ell}_{5}$ & 0 & -1 & -2 & 0 & -2 & 0 & 0 & 3 & 2 & 0
\end{tabular}

The Kähler cone of $\mathcal{A}_{Y^{(3,0)}}$, which is the dual of the Mori cone, is then given by

$$
\begin{aligned}
\mathcal{K}\left(\mathcal{A}_{Y^{(3,0)}}\right) & =\left\{\hat{D} \in \operatorname{Cl}\left(\mathcal{A}_{Y^{(3,0)}}\right) \mid \hat{D} \cdot \hat{\ell}_{i}>0, i=1, \ldots, 5\right\} \\
& =\operatorname{span}\left\{\hat{D}_{1}, \hat{D}_{4}, 2 \hat{D}_{1}+\hat{D}_{6}, 3 \hat{D}_{4}+\hat{D}_{8}, 3 \hat{D}_{2}+3 \hat{D}_{4}+\hat{D}_{8}\right\} .
\end{aligned}
$$

There are a few intersections of coordinate hyperplanes that do intersect in the ambient space, but not on the Calabi-Yau hypersurface $M_{Y^{(3,0)}}$. These are the surfaces $S \in \mathcal{A}_{Y^{(3,0)}}$ with

$$
S \in\left\{\hat{D}_{1} \cap \hat{D}_{7}, \hat{D}_{1} \cap \hat{D}_{8}, \hat{D}_{2} \cap \hat{D}_{8}, \hat{D}_{7} \cap \hat{D}_{9}\right\}
$$

Furthermore, the following intersections $M_{Y^{(3,0)}} \cap C$ with curves $C \in \mathcal{A}_{Y^{(3,0)}}$ are empty:

$$
C \in\left\{\hat{D}_{1} \cap \hat{D}_{3} \cap \hat{D}_{5}, \hat{D}_{1} \cap \hat{D}_{3} \cap \hat{D}_{9}, \hat{D}_{1} \cap \hat{D}_{5} \cap \hat{D}_{9}, \hat{D}_{2} \cap \hat{D}_{3} \cap \hat{D}_{5}, \hat{D}_{3} \cap \hat{D}_{5} \cap \hat{D}_{9}\right\} .
$$

From (2.16) it follows that neither $\hat{\ell}_{4}$ nor $\hat{\ell}_{5}$ can be Mori cone generators of $M_{Y^{(3,0)}}$, because they are contained in $\hat{D}_{3} \cap \hat{D}_{5} \cap \hat{D}_{9}$ and $\hat{D}_{2} \cap \hat{D}_{3} \cap \hat{D}_{5}$, respectively. Systematically eliminating the curves that do not intersect the Calabi-Yau threefold $M_{Y^{(3,0)}}$ leaves us with the Mori cone for $M_{Y^{(3,0)}}$,

\begin{tabular}{c|rrrrrrrrrr}
$\ell \cdot D$ & $x_{1}$ & $x_{2}$ & $x_{3}$ & $x_{4}$ & $x_{5}$ & $x_{6}$ & $x_{7}$ & $x_{8}$ & $x_{9}$ & $K$ \\
\hline$\ell_{1}=\hat{\ell}_{1}$ & 1 & 0 & 0 & 0 & 0 & -2 & 1 & 0 & 3 & -3 \\
$\ell_{2}=\hat{\ell}_{2}$ & 0 & 0 & 1 & 1 & 1 & 0 & 0 & -3 & 0 & 0 \\
$\ell_{3}=\hat{\ell}_{3}$ & 0 & 0 & 1 & 0 & 1 & 1 & -3 & 0 & 0 & 0 \\
$\ell_{4}=\hat{\ell}_{3}+\hat{\ell}_{4}$ & 0 & 1 & 0 & 0 & 0 & 1 & 0 & 0 & -2 & 0 \\
$\ell_{5}=\frac{1}{3}\left(\hat{\ell}_{4}+\hat{\ell}_{5}\right)$ & 0 & 0 & -1 & 0 & -1 & 0 & 1 & 1 & 0 & 0
\end{tabular}

\footnotetext{
${ }^{5}$ We write $\hat{D}_{i}$ for the divisor $\left\{x_{i}=0\right\} \subset \mathcal{A}_{Y^{(3,0)}}$ and $D_{i}$ for the corresponding divisor $\left\{x_{i}=0\right\} \subset M_{Y^{(3,0)}}$.
} 
We next identify the curves generating the Mori cone (corresponding to facets of the Kähler cone) of $M_{Y^{(3,0)}}$. We do this by comparing their Chow cycle with the Chow 1-cycles of the form $D_{i} \cap D_{j} \cap M_{Y^{(3,0)}}$. One finds that

$$
\begin{aligned}
& \ell_{1}=D_{3} \cap D_{6} \cap M_{Y^{(3,0)}}=D_{5} \cap D_{6} \cap M_{Y^{(3,0)}} \\
& \ell_{2}=D_{4} \cap D_{8} \cap M_{Y^{(3,0)}} \\
& \ell_{3}=D_{6} \cap D_{7} \cap M_{Y^{(3,0)}} \\
& \ell_{4}=\frac{1}{3}\left(D_{3} \cap D_{9} \cap M_{Y^{(3,0)}}\right)=\frac{1}{3}\left(D_{5} \cap D_{9} \cap M_{Y^{(3,0)}}\right) \\
& \ell_{5}=D_{3} \cap D_{5} \cap M_{Y^{(3,0)}} .
\end{aligned}
$$

From the Mori cone, we construct the (dual) Kähler cone by finding linear combinations of the generators $D_{1}, \ldots, D_{9}$, such that in the coordinates given by the corresponding columns, we get the five standard basis vectors of $\mathbb{Z}^{5}$. One particular choice allows us to parametrize the Kähler form $\omega(t)$ by $t_{i}=\int_{\ell_{i}} \omega \geq 0$, as

$$
\omega=t_{1} D_{1}+t_{2} D_{4}+t_{3}\left(2 D_{1}-D_{2}+D_{6}\right)+t_{4} D_{2}+t_{5}\left(3 D_{4}+D_{8}\right) .
$$

Note in particular that $t_{5}$ measures the volume of the $\mathbb{P}^{1}$ that resolves the $Y^{3,0}$ to $d P^{0} \times d P^{0}$. In these coordinates, the volume of $M_{Y^{(3,0)}}$ is

$$
\begin{aligned}
\int_{M_{Y^{(3,0)}} \omega^{3}=} & 54 t_{1}^{3}+36 t_{1}^{2} t_{2}+90 t_{1}^{2} t_{3}+243 t_{1}^{2} t_{4}+108 t_{1}^{2} t_{5}+6 t_{1} t_{2}^{2}+36 t_{1} t_{2} t_{3}+ \\
& 108 t_{1} t_{2} t_{4}+36 t_{1} t_{2} t_{5}+48 t_{1} t_{3}^{2}+270 t_{1} t_{3} t_{4}+108 t_{1} t_{3} t_{5}+351 t_{1} t_{4}^{2}+ \\
& 324 t_{1} t_{4} t_{5}+54 t_{1} t_{5}^{2}+3 t_{2}^{2} t_{3}+9 t_{2}^{2} t_{4}+3 t_{2}^{2} t_{5}+9 t_{2} t_{3}^{2}+54 t_{2} t_{3} t_{4}+ \\
& 18 t_{2} t_{3} t_{5}+81 t_{2} t_{4}^{2}+54 t_{2} t_{4} t_{5}+9 t_{2} t_{5}^{2}+8 t_{3}^{3}+72 t_{3}^{2} t_{4}+27 t_{3}^{2} t_{5}+ \\
& 198 t_{3} t_{4}^{2}+162 t_{3} t_{4} t_{5}+27 t_{3} t_{5}^{2}+168 t_{4}^{3}+243 t_{4}^{2} t_{5}+81 t_{4} t_{5}^{2}+9 t_{5}^{3} .
\end{aligned}
$$

The volumes $\operatorname{Vol}\left(D_{i}\right)=\frac{1}{3} \partial_{t_{i}} \operatorname{Vol}\left(Y^{(3,0)}\right)$ of the dual 4-cycles are

$$
\begin{aligned}
\tau_{1}= & 54 t_{1}^{2}+24 t_{1} t_{2}+60 t_{1} t_{3}+162 t_{1} t_{4}+72 t_{1} t_{5}+2 t_{2}^{2}+12 t_{2} t_{3}+36 t_{2} t_{4}+ \\
& 12 t_{2} t_{5}+16 t_{3}^{2}+90 t_{3} t_{4}+36 t_{3} t_{5}+117 t_{4}^{2}+108 t_{4} t_{5}+18 t_{5}^{2} \\
\tau_{2}= & \left(2 t_{1}+t_{3}+3 t_{4}+t_{5}\right)\left(6 t_{1}+2 t_{2}+3 t_{3}+9 t_{4}+3 t_{5}\right) \\
\tau_{3}= & 30 t_{1}^{2}+12 t_{1} t_{2}+32 t_{1} t_{3}+90 t_{1} t_{4}+36 t_{1} t_{5}+t_{2}^{2}+6 t_{2} t_{3}+18 t_{2} t_{4}+ \\
& 6 t_{2} t_{5}+8 t_{3}^{2}+48 t_{3} t_{4}+18 t_{3} t_{5}+66 t_{4}^{2}+54 t_{4} t_{5}+9 t_{5}^{2} \\
\tau_{4}= & 3\left(3 t_{1}+t_{2}+2 t_{3}+4 t_{4}+3 t_{5}\right)\left(9 t_{1}+t_{2}+4 t_{3}+14 t_{4}+3 t_{5}\right) \\
\tau_{5}= & \left(6 t_{1}+t_{2}+3 t_{3}+9 t_{4}+3 t_{5}\right)^{2}
\end{aligned}
$$

We note that all of the nef divisors corresponding to the rays of the Kähler cone have nonzero volume away from the origin of the Kähler cone. In addition, by construction, the divisors $D_{7}$ and $D_{8}$ do shrink on two distinct walls of the Kähler cone. Their volumes are

$$
\operatorname{Vol}\left(D_{7}\right)=\int_{D_{7}} \omega^{2}=t_{3}^{2}, \quad \operatorname{Vol}\left(D_{8}\right)=\int_{D_{8}} \omega^{2}=t_{2}^{2} .
$$

Hence the volumes of the divisors $D_{7}$ and $D_{8}$, respectively, vanish when we shrink the twocycles $\ell_{2}$ and $\ell_{3}$, and the two resulting singularities collide when we set $t_{5}=0$. Nevertheless, the volume of the total Calabi-Yau stays finite as long as $t_{1}>0$. 
The Two Shrinking Divisors. We want to better understand the geometry of the divisors $D_{7}$ and $D_{8}$ on $M_{Y^{(3,0)}}$. Since $M_{Y^{(3,0)}} \subset \mathcal{A}_{Y^{(3,0)}}$ is cut out by a section of the anticanonical bundle, we have to

1. Identify the corresponding divisors $\hat{D}_{7}, \hat{D}_{8} \subset \mathcal{A}_{Y^{(3,0)}}$ of the ambient space as 3dimensional toric varieties.

2. Pull back the anticanonical bundle, $K_{\nabla}$, on $\mathcal{A}_{Y^{(3,0)}}$ to $\hat{D}_{7}, \hat{D}_{8}$.

3. Identify the divisors $D_{7}, D_{8} \subset M_{Y^{(3,0)}}$ as a generic zero section of the pulled-back anticanonical bundle.

The divisor $x_{8}=0$ is particularly simple to describe, so we start with this case. From the fan eq. (2.14) one can easily identify $\hat{D}_{8}=\mathbb{P}^{2} \times \mathbb{P}^{1} \subset \mathcal{A}_{Y^{(3,0)}}$ on the ambient space. In order to pull-back the anticanonical bundle, we note that

$$
-K_{\nabla}=\sum_{i=1}^{9} \mathcal{O}\left(\mathcal{A}_{Y^{(3,0)}}, \hat{D}_{i}\right) \sim 2 \mathcal{O}\left(\mathcal{A}_{Y^{(3,0)}}, \hat{D}_{2}\right)+\mathcal{O}\left(\mathcal{A}_{Y^{(3,0)}}, \hat{D}_{9}\right)
$$

Using this choice of linear equivalence class, one easily finds the pull-back $\left.\left(-K_{\nabla}\right)\right|_{\hat{D}_{8}}=$ $\mathcal{O}_{\mathbb{P}^{2} \times \mathbb{P}^{1}}(0,1)$. A generic section of $\mathcal{O}_{\mathbb{P}^{1}}(1)$ is a single point, therefore

$$
D_{8}=\left\{z=0 \mid z \in H^{0}\left(\hat{D}_{8},\left.\left(-K_{\nabla}\right)\right|_{\hat{D}_{8}}\right)\right\}=\mathbb{P}^{2}
$$

Identifying the other divisor $x_{7}=0$ is somewhat more technical because $\hat{D}_{7}$ is not just a product of projective spaces. However, as with any toric divisor, it is again a toric variety. Here, it turns out to be the variety corresponding to the face of the lattice polytope

$$
\nabla_{7}=\operatorname{conv}\left\{y_{1}, \ldots, y_{5}\right\}=\operatorname{conv}\{(0,0,1),(0,1,0),(-3,2,0),(3,-2,-1),(-2,1,0)\} \text {. }
$$

$\hat{D}_{7}$ is a toric fibration over $\mathbb{P}^{1}$ with generic fiber $\mathbb{P}^{2}$, but its total space is a singular variety. Using eq. (2.26), one then identifies ${ }^{6}$

$$
\left.\left(-K_{\nabla}\right)\right|_{\hat{D}_{7}}=\mathcal{O}\left(\tilde{D}_{5}\right)
$$

It is now easy to identify the toric divisor $V\left(y_{5}\right)$. Again, one finds a smooth projective plane, that is

$$
D_{7}=\hat{D}_{7} \cap Y^{(3,0)}=\tilde{D}_{5}=\mathbb{P}^{2}
$$

$\mathbf{2 . 4}\left(d P_{0}\right)^{3} \rightarrow M_{\left(d P_{0}\right)^{3}}$

In order to embed a $\mathbb{C}^{3} / \mathbb{Z}_{3}$ singularity such that the local sector is placed away from the orientifold plane requires three copies of the $\mathbb{C}^{3} / \mathbb{Z}_{3}$, or $\left(d P_{0}\right)^{3}$. The local singularity is described by the toric diagram in figure 3. A simple ambient toric variety $\mathcal{A}_{\left(d P_{0}\right)^{3}}$ giving

\footnotetext{
${ }^{6}$ We denote the divisors $y_{i}=0$ in $\hat{D}_{7}$ by $\tilde{D}_{i}$.
} 


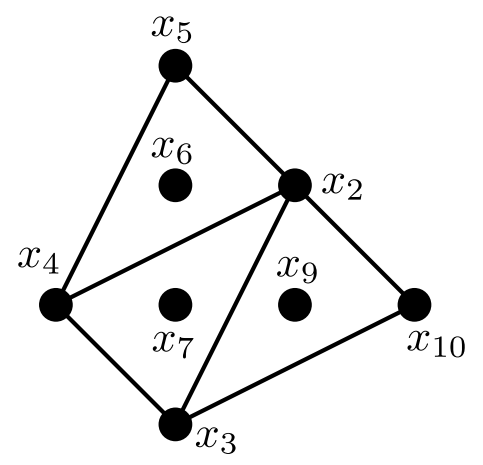

Figure 3. Local model for $\left(d P_{0}\right)^{3}$.

rise to the desired local singularity can be constructed by the relatively simple reflexive polytope with integral points

\begin{tabular}{rrrrrrrrrrrr}
$x_{0}$ & $x_{1}$ & $x_{2}$ & $x_{3}$ & $x_{4}$ & $x_{5}$ & $x_{6}$ & $x_{7}$ & $x_{8}$ & $x_{9}$ & $x_{10}$ & $x_{11}$ \\
\hline 0 & 0 & 1 & 0 & -1 & 0 & 0 & 0 & 0 & 1 & 2 & 1 \\
0 & 0 & 1 & -1 & 0 & 2 & 1 & 0 & 1 & 0 & 0 & 0 \\
-1 & 0 & 2 & 2 & 2 & 2 & 2 & 2 & 1 & 2 & 2 & 1 \\
0 & -1 & 3 & 3 & 3 & 3 & 3 & 3 & 1 & 3 & 3 & 1
\end{tabular}

or equivalently, the following GLSM data:

\begin{tabular}{l|rrrrrrrrrrrrr} 
& $x_{0}$ & $x_{1}$ & $x_{2}$ & $x_{3}$ & $x_{4}$ & $x_{5}$ & $x_{6}$ & $x_{7}$ & $x_{8}$ & $x_{9}$ & $x_{10}$ & $x_{11}$ \\
\hline $\mathbb{C}_{1}^{*}$ & 1 & 0 & 0 & 0 & 0 & 0 & 0 & 0 & 0 & 1 & -2 & 3 \\
$\mathbb{C}_{2}^{*}$ & 0 & 1 & 0 & 0 & 0 & 0 & 0 & 0 & 0 & 0 & 1 & -2 \\
$\mathbb{C}_{3}^{*}$ & 0 & 0 & 1 & 0 & 0 & 0 & 0 & 0 & -1 & 0 & -1 & 1 \\
$\mathbb{C}_{4}^{*}$ & 0 & 0 & 0 & 1 & 0 & 0 & 0 & 0 & 1 & -3 & 2 & -1 \\
$\mathbb{C}_{5}^{*}$ & 0 & 0 & 0 & 0 & 1 & 0 & 0 & 0 & 0 & -3 & 2 & 0 \\
$\mathbb{C}_{6}^{*}$ & 0 & 0 & 0 & 0 & 0 & 1 & 0 & 0 & -2 & 0 & -1 & 2 \\
$\mathbb{C}_{7}^{*}$ & 0 & 0 & 0 & 0 & 0 & 0 & 1 & 0 & -1 & -1 & 0 & 1 \\
$\mathbb{C}_{8}^{*}$ & 0 & 0 & 0 & 0 & 0 & 0 & 0 & 1 & 0 & -2 & 1 & 0
\end{tabular}

The Calabi-Yau hypersurface $M_{\left(d P_{0}\right)^{3}}$ in this ambient toric variety has Hodge numbers $\left(h^{11}, h^{21}\right)=(8,158)$.

This polytope admits 236 different fine star triangulations. Out of these, 30 are compatible with the partial resolution we wish to perform, and out of these 30 we obtain 4 compatible with the $\mathbb{Z}_{2}$ involution structure. One of these triangulations is described by 
the fan below:

$$
\begin{aligned}
\mathcal{F}\left(\mathcal{A}_{\left(d P_{0}\right)^{3}}\right)=\langle & \left\langle x_{0} x_{1} x_{2} x_{8}\right\rangle,\left\langle x_{0} x_{1} x_{2} x_{11}\right\rangle,\left\langle x_{0} x_{1} x_{3} x_{4}\right\rangle,\left\langle x_{0} x_{1} x_{3} x_{11}\right\rangle,\left\langle x_{0} x_{1} x_{4} x_{8}\right\rangle, \\
& \left\langle x_{0} x_{2} x_{3} x_{7}\right\rangle,\left\langle x_{0} x_{2} x_{3} x_{9}\right\rangle,\left\langle x_{0} x_{2} x_{4} x_{6}\right\rangle,\left\langle x_{0} x_{2} x_{4} x_{7}\right\rangle,\left\langle x_{0} x_{2} x_{5} x_{6}\right\rangle, \\
& \left\langle x_{0} x_{2} x_{5} x_{8}\right\rangle,\left\langle x_{0} x_{2} x_{9} x_{10}\right\rangle,\left\langle x_{0} x_{2} x_{10} x_{11}\right\rangle,\left\langle x_{0} x_{3} x_{4} x_{7}\right\rangle,\left\langle x_{0} x_{3} x_{9} x_{10}\right\rangle, \\
& \left\langle x_{0} x_{3} x_{10} x_{11}\right\rangle,\left\langle x_{0} x_{4} x_{5} x_{6}\right\rangle,\left\langle x_{0} x_{4} x_{5} x_{8}\right\rangle,\left\langle x_{1} x_{2} x_{3} x_{7}\right\rangle,\left\langle x_{1} x_{2} x_{3} x_{9}\right\rangle, \\
& \left\langle x_{1} x_{2} x_{4} x_{6}\right\rangle,\left\langle x_{1} x_{2} x_{4} x_{7}\right\rangle,\left\langle x_{1} x_{2} x_{6} x_{8}\right\rangle,\left\langle x_{1} x_{2} x_{9} x_{11}\right\rangle,\left\langle x_{1} x_{3} x_{4} x_{7}\right\rangle, \\
& \left\langle x_{1} x_{3} x_{9} x_{11}\right\rangle,\left\langle x_{1} x_{4} x_{6} x_{8}\right\rangle,\left\langle x_{2} x_{5} x_{6} x_{8}\right\rangle,\left\langle x_{2} x_{9} x_{10} x_{11}\right\rangle,\left\langle x_{3} x_{9} x_{10} x_{11}\right\rangle, \\
& \left.\left\langle x_{4} x_{5} x_{6} x_{8}\right\rangle\right\rangle
\end{aligned}
$$

corresponding to the following Stanley-Reisner ideal:

$$
\begin{aligned}
S R\left(\mathcal{A}_{\left(d P_{0}\right)^{3}}\right)= & \left\langle x_{1} x_{5}, x_{1} x_{10}, x_{3} x_{8}, x_{7} x_{8}, x_{8} x_{9}, x_{8} x_{10}, x_{8} x_{11}, x_{4} x_{11}, x_{5} x_{11},\right. \\
& x_{6} x_{11}, x_{7} x_{11}, x_{3} x_{5}, x_{3} x_{6}, x_{4} x_{9}, x_{4} x_{10}, x_{5} x_{7}, x_{6} x_{7}, \\
& x_{7} x_{9}, x_{7} x_{10}, x_{5} x_{9}, x_{6} x_{9}, x_{6} x_{10}, x_{5} x_{10}, x_{0} x_{1} x_{6}, x_{0} x_{1} x_{7}, \\
& x_{0} x_{1} x_{9}, x_{0} x_{6} x_{8}, x_{2} x_{4} x_{8}, x_{0} x_{9} x_{11}, x_{2} x_{3} x_{11}, x_{2} x_{3} x_{4}, \\
& \left.x_{2} x_{3} x_{10}, x_{2} x_{4} x_{5}, x_{0} x_{1} x_{2} x_{3}, x_{0} x_{1} x_{2} x_{4}\right\rangle .
\end{aligned}
$$

The Mori cone of the ambient space is then given by:

\begin{tabular}{l|rrrrrrrrrrrrr} 
& $x_{0}$ & $x_{1}$ & $x_{2}$ & $x_{3}$ & $x_{4}$ & $x_{5}$ & $x_{6}$ & $x_{7}$ & $x_{8}$ & $x_{9}$ & $x_{10}$ & $x_{11}$ & $K$ \\
\hline$\hat{\ell}_{1}$ & 0 & 0 & 1 & 1 & 1 & 0 & 0 & -3 & 0 & 0 & 0 & 0 & 0 \\
$\hat{\ell}_{2}$ & 0 & 0 & -1 & 0 & -1 & 0 & 1 & 1 & 0 & 0 & 0 & 0 & 0 \\
$\hat{\ell}_{3}$ & 1 & 0 & 0 & 0 & 0 & -2 & 1 & 0 & 3 & 0 & 0 & 0 & -3 \\
$\hat{\ell}_{4}$ & 0 & -1 & 1 & 1 & 0 & 0 & 0 & 0 & 0 & -3 & 0 & 2 & 0 \\
$\hat{\ell}_{5}$ & 0 & 1 & 0 & 0 & 0 & 0 & 0 & 0 & 0 & 0 & 1 & -2 & 0 \\
$\hat{\ell}_{6}$ & 0 & 0 & -1 & -1 & 0 & 0 & 0 & 1 & 0 & 1 & 0 & 0 & 0 \\
$\hat{\ell}_{7}$ & 0 & 1 & 0 & 0 & 0 & 1 & 0 & 0 & -2 & 0 & 0 & 0 & 0 \\
$\hat{\ell}_{8}$ & 0 & -1 & 1 & 0 & 1 & 0 & -3 & 0 & 2 & 0 & 0 & 0 & 0 \\
$\hat{\ell}_{9}$ & 1 & 0 & 0 & 0 & 0 & 0 & 0 & 0 & 0 & 1 & -2 & 3 & -3
\end{tabular}

Notice that, while the Mori cone itself is 8-dimensional, we need 9 generators in order to span the whole cone of effective curves. In other words, the Mori cone is not a simplicial cone.

Some of the curves in the Mori cone for $\mathcal{A}_{\left(d P_{0}\right)^{3}}$ are not contained in the Calabi-Yau hypersurface $M_{\left(d P_{0}\right)^{3}}$, and in order to obtain the Mori cone for the Calabi-Yau hypersurface we need to eliminate these curves. The curves actually contained in $M_{\left(d P_{0}\right)^{3}}$ then generate a smaller cone, which often equals the Mori cone of the hypersurface. An observation that is useful in order to do this systematically is that:

$$
-K_{\nabla} \sim 3 \mathcal{O}\left(\mathcal{A}_{\left(d P_{0}\right)^{3}}, \hat{D}_{0}\right) .
$$

From this fact we can easily obtain which curves are not in the Calabi-Yau hypersurface. For instance, since $\hat{\ell}_{4}$ is contained in $\hat{D}_{1} \cap \hat{D}_{9}$, and $x_{0} x_{1} x_{9}$ is in the Stanley-Reisner 
ideal (2.34), $\hat{\ell}_{4}$ is not contained in the Calabi-Yau hypersurface. Proceeding systematically, one finds

\begin{tabular}{crrrrrrrrrrrrrr} 
& $\ell \cdot D$ & $x_{0}$ & $x_{1}$ & $x_{2}$ & $x_{3}$ & $x_{4}$ & $x_{5}$ & $x_{6}$ & $x_{7}$ & $x_{8}$ & $x_{9}$ & $x_{10}$ & $x_{11}$ & $K$ \\
\hline$\ell_{1}=$ & 0 & 0 & 1 & 1 & 1 & 0 & 0 & -3 & 0 & 0 & 0 & 0 & 0 \\
$\ell_{2}=$ & $\hat{\ell}_{1}$ & 0 & 0 & -1 & 0 & -1 & 0 & 1 & 1 & 0 & 0 & 0 & 0 & 0 \\
$\ell_{3}=$ & $\hat{\ell}_{2}$ & 1 & 0 & 0 & 0 & 0 & -2 & 1 & 0 & 3 & 0 & 0 & 0 & -3 \\
$\ell_{4}=$ & $\hat{\ell}_{3}$ & 0 & 0 & 1 & 1 & 0 & 0 & 0 & 0 & 0 & -3 & 1 & 0 & 0 \\
$\ell_{5}=$ & $\hat{\ell}_{4}+\hat{\ell}_{5}$ & 0 & 1 & 0 & 0 & 0 & 0 & 0 & 0 & 0 & 0 & 1 & -2 & 0 \\
$\ell_{6}=$ & $\hat{\ell}_{5}$ & 0 & 0 & -1 & -1 & 0 & 0 & 0 & 1 & 0 & 1 & 0 & 0 & 0 \\
$\ell_{7}=$ & $\hat{\ell}_{6}$ & 0 & 1 & 0 & 0 & 0 & 1 & 0 & 0 & -2 & 0 & 0 & 0 & 0 \\
$\ell_{8}=$ & $\hat{\ell}_{7}$ & 0 & 0 & 1 & 0 & 1 & 1 & -3 & 0 & 0 & 0 & 0 & 0 & 0 \\
$\ell_{9}=$ & $\hat{\ell}_{7}+\hat{\ell}_{8}$ & 1 & 0 & 0 & 0 & 0 & 0 & 0 & 0 & 0 & 1 & -2 & 3 & -3 \\
$\ell_{10}=\frac{1}{2}\left(\hat{\ell}_{4}+\hat{\ell}_{8}+3 \hat{\ell}_{6}+3 \hat{\ell}_{2}+2 \hat{\ell}_{1}\right)$ & 0 & -1 & -1 & 0 & 0 & 0 & 0 & 0 & 1 & 0 & 0 & 1 & 0
\end{tabular}

The 8-dimensional Mori cone of the hypersurface has 10 rays, so it is even less simplicial than the Mori cone of the ambient space. Its dual, the Kähler cone of the Calabi-Yau threefold $M_{\left(d P_{0}\right)^{3}}$, is an 8-dimensional cone generated by 29 rays!

To make the discussion more manageable, we will restrict ourselves to the 5-dimensional subspace

$$
\operatorname{span}\left\{D_{0}, D_{1}, D_{2}, D_{3}+D_{4}, D_{7}\right\}
$$

of the 8-dimensional Kähler moduli space. In section 4.3, this will turn out to be the $h_{+}^{11}\left(M_{\left(d P_{0}\right)^{3}}\right)=5$-dimensional subspace of orientifold-invariant Kähler moduli, but for the purposes of this section we can just take it to be a simplifying assumption. When intersected with this subspace, the Kähler cone is generated by the 8 divisor classes

$$
\begin{aligned}
& \omega \in \operatorname{span}_{\mathbb{R}_{\geq}\{}\left\{D_{0}, D_{1}-D_{2}+\left(D_{3}+D_{4}\right), D_{1}-D_{2}+2\left(D_{3}+D_{4}\right)+D_{7},\right. \\
& 3 D_{1}-3 D_{2}+3\left(D_{3}+D_{4}\right)+D_{7},-3 D_{2}+3\left(D_{3}+D_{4}\right)+D_{7}, \\
&\left.-D_{2}+\left(D_{3}+D_{4}\right),-D_{2}+2\left(D_{3}+D_{4}\right)+D_{7}\right\}
\end{aligned}
$$

A particular patch of the (non-simplicial) Kähler cone that will be useful in the following can be parametrized as

$$
\begin{gathered}
\omega=t_{0} D_{0}+t_{1}\left(D_{1}-D_{2}+\left(D_{3}+D_{4}\right)\right)+t_{2}\left(D_{1}-D_{2}+2\left(D_{3}+D_{4}\right)+D_{7}\right)+ \\
t_{3}\left(3 D_{1}-3 D_{2}+3\left(D_{3}+D_{4}\right)+D_{7}\right)+t_{4}\left(-3 D_{2}+3\left(D_{3}+D_{4}\right)+D_{7}\right)
\end{gathered}
$$

With respect to this Kähler class, the volume of the invariant divisors $D_{7}$ and $D_{6}+D_{9} \simeq$ $D_{0}-3\left(D_{3}+D_{4}\right)-2 D 7$ is

$$
\operatorname{Vol}\left(D_{7}\right)=\int_{D_{7}} \omega^{2}=t_{1}^{2}, \quad \operatorname{Vol}\left(D_{6}+D_{9}\right)=\int_{D_{6}+d_{9}} \omega^{2}=2 t_{2}^{2}
$$


and the volume of the Calabi-Yau is

$$
\begin{aligned}
\operatorname{Vol}\left(M_{\left(d P_{0}\right)^{3}}\right)= & \int_{M_{\left(d P_{0}\right)^{3}}} \omega^{3} \\
= & 36 t_{0}^{3}+198 t_{0}^{2} t_{1}+330 t_{0} t_{1}^{2}+183 t_{1}^{3}+234 t_{0}^{2} t_{2}+792 t_{0} t_{1} t_{2}+660 t_{1}^{2} t_{2} \\
& +474 t_{0} t_{2}^{2}+792 t_{1} t_{2}^{2}+316 t_{2}^{3}+594 t_{0}^{2} t_{3}+1980 t_{0} t_{1} t_{3}+1650 t_{1}^{2} t_{3} \\
& +2376 t_{0} t_{2} t_{3}+3960 t_{1} t_{2} t_{3}+2376 t_{2}^{2} t_{3}+2970 t_{0} t_{3}^{2}+4950 t_{1} t_{3}^{2} \\
& +5940 t_{2} t_{3}^{2}+4950 t_{3}^{3}+108 t_{0}^{2} t_{4}+360 t_{0} t_{1} t_{4}+300 t_{1}^{2} t_{4}+432 t_{0} t_{2} t_{4} \\
& +720 t_{1} t_{2} t_{4}+432 t_{2}^{2} t_{4}+1080 t_{0} t_{3} t_{4}+1800 t_{1} t_{3} t_{4}+2160 t_{2} t_{3} t_{4} \\
& +2700 t_{3}^{2} t_{4}+54 t_{0} t_{4}^{2}+90 t_{1} t_{4}^{2}+108 t_{2} t_{4}^{2}+270 t_{3} t_{4}^{2}+9 t_{4}^{3} .
\end{aligned}
$$

Hence, at $t_{1}=0$ the $d P_{0}$ surface $D_{7}$ shrinks and at $t_{2}=0$ the two $d P_{0}$ surfaces $D_{6}$ and $D_{9}$ shrink simultaneously, see figure 3. Furthermore, the toric curves $D_{2} \cap D_{3}$ and $D_{2} \cap D_{4}$ separate $D_{7}$ from $D_{9}$ and $D_{7}$ from $D_{6}$, respectively. Their volume is

$$
\frac{1}{2} \operatorname{Vol}\left(D_{2} \cap\left(D_{3}+D_{4}\right)\right)=t_{3}+t_{4} .
$$

Therefore, if we set $t_{1}=t_{2}=t_{3}=t_{4}=0$, the three $\mathbb{C}^{3} / \mathbb{Z}_{3}$ singularities from shrinking $D_{6}, D_{7}$, and $D_{9}$ collide and produce an enhanced (non-orbifold) singularity. As long as $t_{0}$ stays finite, the Calabi-Yau threefold is of finite volume in these limits.

\section{Adding D-branes to the model}

So far we have only discussed the closed string sector, but realistic models also require the specification of an open string sector. There are two main ingredients in the open string sector of type IIB compactifications: D-branes giving the gauge dynamics, and orientifolds canceling the tadpoles. We will postpone the discussion of orientifolds to section 4, and deal with the systematic incorporation of D-branes in this section.

We will assume that we have a consistent (i.e., tadpole-free) local model of branes at singularities, described in terms of a quiver gauge theory. In order to view this local model as part of a global model we need to give a description of the fractional branes and flavor branes in terms of the geometry of the global model. In order to keep our discussion concrete, we will focus on a particular model on the $\mathbb{C}^{3} / \mathbb{Z}_{3}$ singularity.

The $\mathbb{C}^{3} / \mathbb{Z}_{3}$ MSSM. The model that we will use to illustrate our discussion was originally introduced in [4] as a toy model for the MSSM. We reproduce it in figure 4. Notice that in this quiver we have both gauge groups and flavor groups. The gauge groups are obtained by introducing fractional branes on the $\mathbb{C}^{3} / \mathbb{Z}_{3}$ singularity. In order to analyze the physics of branes at this singularity, it is convenient to have the dimer model for $\mathbb{C}^{3} / \mathbb{Z}_{3}$. This is given by the honeycomb periodic lattice, shown in figure 5 .

Despite focusing on the previous example - which is all we need for our chosen toy models $\left(d P_{0}\right)^{n}$ — we will formulate the discussion in general terms, and it will be applicable to any singularity with at least one contracting 4-cycle. Models with no contracting 4-cycles 


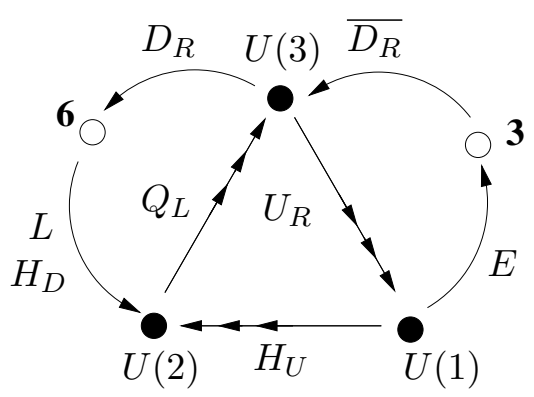

Figure 4. A toy model for the MSSM, from [4]. The filled dark dots denote gauge groups, while the white dots denote global symmetry groups, coming from non-compact $D 7$ branes. The labels on the arrows denote with which MSSM field they should be identified.

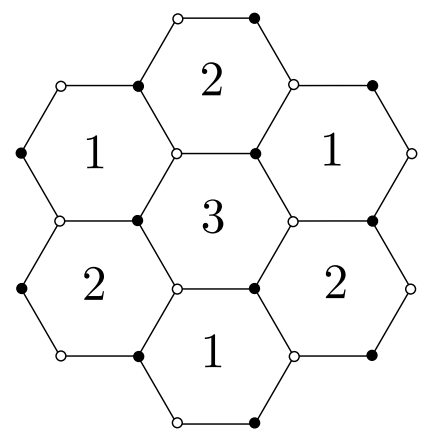

Figure 5. Dimer model for branes at the $\mathbb{C}^{3} / \mathbb{Z}_{3}$ singularity, given by the periodic honeycomb lattice (we have only shown a few cells). The labels on the faces of the dimer model indicate which gauge factor in figure 4 they correspond to.

$\left(L^{a, b, a}\right.$ geometries, or $\left.\mathbb{C}^{3} /\left(\mathbb{Z}_{2} \times \mathbb{Z}_{2}\right)\right)$ can also be interesting for model building purposes, see for example [36, 37]. They cannot support standard-model-like structures, though, so we omit their detailed description, although it should be possible to give a description quite similar to the one below. In the context of the Toric Lego such supersymmetry breaking sectors can be incorporated into a GMSB model [7], and the total model can then be analyzed using the techniques below.

We review the large volume description of gauge branes in section 3.1. This construction is well known in the literature [38-47]. We will follow the convention of describing the branes by elements in the derived category of coherent sheaves. This description differs from the more conventional one in physics (a brane with a vector bundle on top) by some subtleties that will not matter much in our analysis, except for a factor of $\sqrt{K_{S}^{\vee}}$ relating the sheaf and the bundle [48,49], with $K_{S}$ the canonical class of the divisor wrapped by the brane. Accordingly, we will distinguish the sheaf $\mathcal{E}$ from its associated vector bundle $F_{\mathcal{E}}=\mathcal{E} \otimes \sqrt{K_{S}^{\vee}} \cdot{ }^{7}$

\footnotetext{
${ }^{7}$ When $S$ is not $\operatorname{Spin}$ we have that $\mathcal{F}_{\mathcal{E}}$ is not an honest bundle. Nevertheless all the formulas for physical quantities are well defined, as it is manifest from the sheaf description of the brane. This is one reason why we will prefer the "sheafy" description.
} 
To our knowledge a similar systematic dictionary between the quiver and large volume languages has not been given for the kind of flavor branes and singularities that we are interested in. We give the first steps in this direction in section 3.2, by obtaining some of the charges of the flavor branes by imposing the right quiver structure. It would certainly be interesting to give a general and complete dictionary for flavor branes at the same level as that for gauge branes, but we do not attempt to do so in this paper.

\subsection{Blowing up the fractional branes}

Space-time filling D-branes on a IIB compactification on a smooth Calabi-Yau $X$ are described by objects in the bounded derived category of coherent sheaves on $X$, generally denoted by $D^{b}(X)$. We will not review this here, and instead refer the reader to [43] for a nice review. This category can be thought of as the set of branes in the B-model. As such, it does not depend on where we are in Kähler moduli space, and in particular it can also describe branes at the singular quiver point.

Such a description is not necessarily the most suitable one at every point in moduli space. Close to orbifold points, for example, a more convenient description of D-brane states can be given in terms of quiver representations, and in the toric case dimer models. The information describing the D-brane is in this case encoded in the ranks of the gauge nodes, and the vevs of the bifundamentals. Nevertheless, as mentioned above, the categorical description of branes is valid everywhere in moduli space, and thus one expects an equivalence between the respective categories. In particular cases such a correspondence can be proven, one of the most celebrated such results being the one by Bridgeland, King and Reid [50], which states that:

$$
D_{G}(M)=D^{b}(\widetilde{M / G})
$$

with $M$ typically $\mathbb{C}^{3}$ in physical applications, and $G$ a finite subgroup of $\mathrm{SU}(d), d=$ $\operatorname{dim}(M)$. The term of the left represents the branes in $\mathbb{C}^{3}$ together with an action under $G$ (an equivariant structure), i.e., the ordinary description of branes on orbifolds, while the term on the right represents the category of coherent sheaves on the resolution of the orbifold $M / G$.

We will be interested in continuing the quiver description to large volume in cases where the singularity is not of orbifold type, and not necessarily a contracting del Pezzo surface either. In this case there are only partial results, the most useful for us being the method proposed in [47], which we proceed to review now.

\subsubsection{The $\Psi$ map}

Let us start by describing in terms of the global geometry the fractional branes giving the gauge group. That is, we want to obtain the exceptional collection that describes the fractional branes at large volume. The basic technology we need for doing this was described in [47]. Notice that the exceptional collection for a local $\mathbb{C}^{3} / \mathbb{Z}_{3}$ singularity is well-known, and has been obtained by other methods [38, 40-43]. In particular it is an orbifold, and as such falls into the class studied by [50], who give the explicit functor 

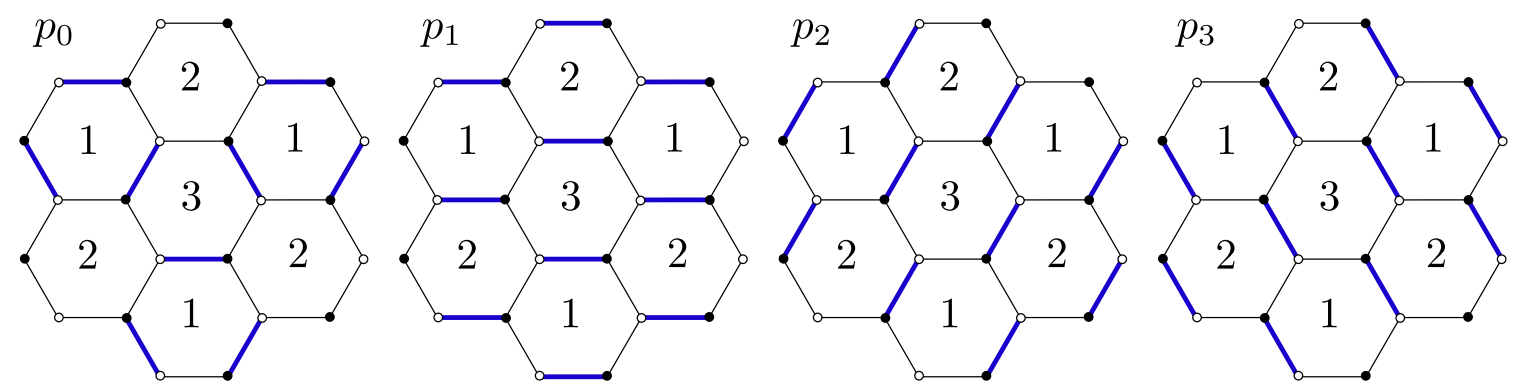

Figure 6. Relevant perfect matchings for the $\mathbb{C}^{3} / \mathbb{Z}_{3}$ dimer model. We have omitted one of the internal perfect matchings.

between the large volume and quiver categories. We have chosen to compute the basis of fractional branes using the $\Psi$ map [47] since this method works for any toric singularity with a compact 4-cycle.

The first step in constructing the $\Psi$ map consists of determining the perfect matchings of the dimer model, and their interpretation in terms of divisors of the local geometry. There are 5 perfect matchings of the honeycomb lattice in figure 5 , of which 2 are reference matchings - associated with the interior point of the toric diagram, or equivalently with the compact $\mathbb{P}^{2}$ - and the other three correspond to the exterior points of the toric diagram. We show this structure in figure 6.

From the perfect matchings in figure 6 we can reconstruct the geometry, as explained in [51-53]. In particular, to each external perfect matching we can associate a non-compact divisor of the $\mathbb{C}^{3} / \mathbb{Z}_{3}$ geometry, i.e., the $D_{1}, D_{2}, D_{3}$ divisors in figure 1 . This is done as follows: notice that each edge in the dimer has a natural orientation (going from the white node to the black node, for example). Given this orientation, it makes sense to consider $p_{i}-p_{0}$ as a closed oriented cycle in the dimer. The cycle is obtained by superposing the edges belonging to $p_{i}$ with the edges belonging to $p_{0}$, with the understanding that edges belonging to both perfect matchings "annihilate" each other. This operation defines a $(p, q)$ cycle on the $T^{2}$ where the dimer model is defined. Taking this $(p, q)$ as a point in the integer lattice, the convex hull of the resulting set of points turns out to be the diagram for the toric geometry giving rise to the dimer. For our particular example, we obtain the following winding numbers:

$$
p_{0}-p_{0}=(0,0) \quad p_{1}-p_{0}=(1,1) \quad p_{2}-p_{0}=(-1,0) \quad p_{3}-p_{0}=(0,-1) .
$$

By comparison with figure 1 we thus obtain the following identification between perfect matchings and external divisors:

$$
\left(p_{0}, p_{1}, p_{2}, p_{3}\right) \sim\left(D_{6}, D_{3}, D_{2}, D_{1}\right)
$$

We can now proceed to describe the exceptional collection obtained from the $\Psi$ map in our particular example. Let us take as our reference fractional brane the gauge factor denoted by " 1 " in figure 5 . In order to obtain the exceptional collection we construct an 


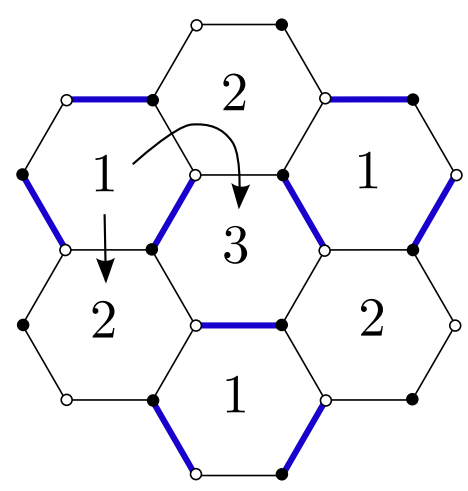

Figure 7. Allowed open paths giving the elements of the exceptional collection associated to the Beilinson quiver for $\mathbb{C}^{3} / \mathbb{Z}_{3}$, taking $p_{0}$ as a reference matching.

open (allowed [47]) path in the dimer from 1 to 2 and another from 1 to 3 . The paths we have chosen are depicted in figure 7. The exceptional collection giving the Beilinson quiver is then determined by the crossing of the open paths with the perfect matchings. In our conventions, and taking into account the relation (3.3) between perfect matchings and divisors we obtain: ${ }^{8}$

$$
P=\left(\mathcal{O}_{\mathbb{P}^{2}}, \mathcal{O}_{\mathbb{P}^{2}}\left(D_{3}\right), \mathcal{O}_{\mathbb{P}^{2}}\left(D_{3}+D_{1}\right)\right)
$$

Taking into account linear equivalence of divisors on the $\mathbb{P}^{2}$, and simplifying notation a bit, we end up with:

$$
P=(\mathcal{O}, \mathcal{O}(1), \mathcal{O}(2))
$$

which is a well-known projective basis for the derived category of branes on $\mathbb{P}^{2}$.

In order to identify the physical fractional branes from the collection (3.5) we still need to work a little bit more, and dualize $P .{ }^{9}$ That is, the collection that describes the physical fractional branes is not (3.5), but rather the following related collection:

$$
\mathcal{E}=\left(\mathcal{E}_{1}, \mathcal{E}_{2}, \mathcal{E}_{3}\right)
$$

Constructing the objects of the collection $\mathcal{E}$ is non-trivial, but thankfully their Chern character is much more easily computed. We have the basic relation:

$$
\operatorname{ch}\left(\mathcal{E}_{i}\right)=\left(S^{-1}\right)_{j i} \operatorname{ch}\left(P_{j}\right) .
$$

\footnotetext{
${ }^{8}$ Our notation departs slightly from the conventions in $[44,46,47]$. We denote the collection (3.4) $P=\left\{P_{i}\right\}$ since it is a collection of projective objects in the del Pezzo case [45], and we will reserve the symbol $\mathcal{E}_{i}$ for the fractional branes in $\mathbb{C}^{3} / \mathbb{Z}_{3}$ themselves. $\mathcal{E}_{i}^{\vee}$ will then denote the dual of the sheaf $\mathcal{E}_{i}$ in the ordinary sense (for example $c_{i}\left(\mathcal{E}^{\vee}\right)=(-1)^{i} c_{i}(\mathcal{E})$ ).

${ }^{9}$ Technically, what we are doing here is constructing the fractional branes in terms of projective objects by mutation, and taking Chern characters. We refer the reader interested in the details to the nice exposition in $[45]$.
} 
The matrix $S$ is defined as follows:

$$
S_{i j}=\operatorname{dim} \operatorname{Hom}\left(\mathcal{E}_{i}, \mathcal{E}_{j}\right)=H^{0}\left(\mathbb{P}^{2}, \mathcal{E}_{i}^{\vee} \otimes \mathcal{E}_{j}\right) .
$$

Since $\mathcal{E}_{i}$ are line bundles on a toric variety, it is easy to compute $S$ just by counting sections:

$$
S=\left(\begin{array}{lll}
1 & 3 & 6 \\
0 & 1 & 3 \\
0 & 0 & 1
\end{array}\right)
$$

The inverse matrix $S^{-1}$ is thus given by:

$$
S^{-1}=\left(\begin{array}{ccc}
1 & -3 & 3 \\
0 & 1 & -3 \\
0 & 0 & 1
\end{array}\right)
$$

Plugging this matrix into (3.7), we obtain:

$$
\begin{aligned}
& \operatorname{ch}\left(\mathcal{E}_{1}^{\vee}\right)=1 \\
& \operatorname{ch}\left(\mathcal{E}_{2}^{\vee}\right)=-2+H+\frac{1}{2} H^{2} \\
& \operatorname{ch}\left(\mathcal{E}_{3}^{\vee}\right)=1-H+\frac{1}{2} H^{2} .
\end{aligned}
$$

A simple check of this expression is that if we add up the Chern characters of the three D-branes we get $H^{2}$. Due to the Chern-Simons coupling on the worldvolume of the branes, we have that this induces precisely one unit of D3-brane charge (there are also well known curvature contributions to the charge, but they do not contribute here). This agrees with the expected result that the quiver with all ranks equal represents the theory on a single $D 3$ probing the singularity.

In our particular case we want to introduce fractional branes of different ranks, in particular we want to consider the object:

$$
\mathcal{E}_{M S S M}=\mathcal{E}_{1}+2 \mathcal{E}_{2}+3 \mathcal{E}_{3}
$$

The Chern-Simons coupling for a $D 7$-brane described by a sheaf $\mathcal{E}$ wrapping a divisor $S$ is given by:

$$
S_{C . S .}=\int_{C \times \mathbb{R}^{3,1}} C^{(R R)} \wedge \operatorname{ch}(\mathcal{E}) \wedge \sqrt{\frac{\operatorname{Td}(T S)}{\operatorname{Td}(N S)}},
$$

with $C^{(R R)}=C_{0}+C_{2}+\ldots$ the formal sum of $R R$ fields, and $T S, N S$ refer to the tangent and normal bundles of $S$ in the Calabi-Yau $X$. The Todd class $\operatorname{Td}(T S)$ of $S$ is defined as follows:

$$
\operatorname{Td}(T S)=1+\frac{1}{2} c_{1}(T S)+\frac{c_{1}(T S)^{2}+c_{2}(T S)}{12}
$$


For example, in the particular case of $\mathbb{P}^{2}$, we have:

$$
\operatorname{Td}\left(T \mathbb{P}^{2}\right)=1+\frac{3}{2} H+H^{2},
$$

with $H$ the hyperplane class.

We can encode this charge information into the charge vector:

$$
\Gamma_{\mathcal{E}}=[S] \operatorname{ch}(\mathcal{E}) \sqrt{\frac{\operatorname{Td}(T S)}{\operatorname{Td}(N S)}},
$$

with $[S]$ the class Poincare dual to $S$. In what follows we will often forget the distinction between cohomology classes and divisors, in order to lighten the notation, and we will omit the space-time part of the charge.

Computing the induced $D 5$ and $D 3$ charges from (3.16), (3.11) and the facts that $\left.N S\right|_{S}=\left.D_{S}\right|_{S}=\mathcal{O}(-3 H), c(T S)=(1+H)^{3}$ :

$$
\begin{aligned}
& \Gamma_{\mathcal{E}_{1}}=[S] \wedge\left(1+\frac{3}{2} H+\frac{5}{4} H^{2}\right) \\
& \Gamma_{\mathcal{E}_{2}}=[S] \wedge\left(-2-2 H-\frac{1}{2} H^{2}\right) \\
& \Gamma_{\mathcal{E}_{3}}=[S] \wedge\left(1+\frac{1}{2} H+\frac{1}{4} H^{2}\right),
\end{aligned}
$$

so we get the total charge:

$$
\begin{aligned}
\Gamma_{\mathcal{E}_{M S S M}} & =\Gamma_{\mathcal{E}_{1}}+2 \Gamma_{\mathcal{E}_{2}}+3 \Gamma_{\mathcal{E}_{3}} \\
& =[S] \wedge\left(H+H^{2}\right) .
\end{aligned}
$$

We see that we have a total non-vanishing induced $D_{5}$ charge, and also the expected induced $D 3$ charge. The induced $D_{5}$ charge signals a tadpole, since it is supported on a 2-cycle with a compact dual cycle given in this case by the $\mathbb{P}^{2}$ itself. We need to cancel this tadpole by introducing extra ingredients into our configuration in the form of flavor D7 branes. We will do this in section 3.2 .

A subtlety in the definition of the $D 3$ charge. In the discussion above we have taken the usual conventions in the exceptional collection literature. Unfortunately there is a subtlety that is important in physics applications of these formulas: what we ordinarily call a $D 3$ brane (defined as the object mutually supersymmetric with respect to a large volume $D 7$ brane) has

$$
\Gamma_{D 3}=-[p t],
$$

with $[p t]$ the class of a point in the Calabi-Yau, instead of $\Gamma_{D 3}=[p t]$, as one may guess at first. An easy way to show this is by noticing that the expression for the central charge of a $D 7$ with support on $S$ at large volume, given by:

$$
Z(D 7)=\int_{X} e^{-(B+i J)} \wedge \Gamma \sim-\frac{1}{2} \int_{S} J \wedge J
$$


is a large negative number. Taking the sign as in (3.19) gives a central charge with the same sign:

$$
Z(D 3)=\int_{X} e^{-(B+i J)} \wedge(-[p t])=-1,
$$

and thus both objects preserve the same supersymmetry. ${ }^{10}$

The simplest way of taking this issue into account is simply to multiply by -1 the Chern characters that we found above for the fractional branes. This does not change the quiver, but now the charges of the fractional branes add up to minus the class of a point. The Chern characters for the objects in the exceptional collection are thus given by:

$$
\begin{aligned}
& \operatorname{ch}\left(\mathcal{E}_{1}\right)=-1 \\
& \operatorname{ch}\left(\mathcal{E}_{2}\right)=2-H-\frac{1}{2} H^{2} \\
& \operatorname{ch}\left(\mathcal{E}_{3}\right)=-1+H-\frac{1}{2} H^{2} .
\end{aligned}
$$

and the corresponding charges by:

$$
\begin{aligned}
& \Gamma_{\mathcal{E}_{1}}=[S] \wedge\left(-1-\frac{3}{2} H-\frac{5}{4} H^{2}\right) \\
& \Gamma_{\mathcal{E}_{2}}=[S] \wedge\left(2+2 H+\frac{1}{2} H^{2}\right) \\
& \Gamma_{\mathcal{E}_{3}}=[S] \wedge\left(-1-\frac{1}{2} H-\frac{1}{4} H^{2}\right) .
\end{aligned}
$$

\subsubsection{Computing the spectrum}

Given two branes, described by sheaves $\mathcal{E}, \mathcal{F}$ on the divisors $S$ and $T$, embedded on the Calabi-Yau by the maps $i: S \hookrightarrow X, j: T \hookrightarrow X$ respectively, we have that the spectrum of strings between them is expected to be counted by [49]:

$$
\operatorname{Ext}^{\bullet}\left(i_{*} \mathcal{E}, j_{*} \mathcal{F}\right) .
$$

Ext groups are typically hard to compute, but luckily in the cases that we are dealing with the calculation simplifies. For two $D 7$ branes wrapping the same divisor, one has that [45]:

$$
\operatorname{Ext}_{X}^{i}\left(i_{*} \mathcal{E}, j_{*} \mathcal{F}\right)=\operatorname{Ext}_{S}^{i}(\mathcal{E}, \mathcal{F}) \oplus \operatorname{Ext}_{S}^{3-i}(\mathcal{F}, \mathcal{E})
$$

If one is just interested in computing indices the calculation simplifies further:

$$
\begin{aligned}
\sum(-1)^{i} \operatorname{dim} \operatorname{Ext}_{X}^{i}\left(i_{*} \mathcal{E}, j_{*} \mathcal{F}\right) & =\sum(-1)^{i} \operatorname{dim} \operatorname{Ext}_{S}^{i}(\mathcal{E}, \mathcal{F})-\sum(-1)^{i} \operatorname{dim} \operatorname{Ext}_{S}^{i}(\mathcal{F}, \mathcal{E}) \\
& =\chi(\mathcal{E}, \mathcal{F})-\chi(\mathcal{F}, \mathcal{E}) .
\end{aligned}
$$

\footnotetext{
${ }^{10}$ The negative sign also follows from duality and a familiar fact in the heterotic string: in order to cancel the heterotic tadpole in a $K 3 \times T^{2}$ compactification without switching instantons on the gauge bundle, one needs to introduce 24 mobile $N S 5$ s. Dualizing to F-theory, these branes appear as 24 mobile D3s. The D3 tadpole created by these branes is canceled by the $D 3$ charge induced by the curvature couplings of the 24 7-branes wrapping the base $K 3$ in F-theory. Choosing the (standard) Chern-Simons coupling for D7s as in (3.13) then forces us to set $\Gamma_{D 3}=-[p t]$.
} 
where we have used Hirzebruch-Riemann-Roch in the last line, and $\chi(\mathcal{E}, \mathcal{F})$ is defined as:

$$
\chi(\mathcal{E}, \mathcal{F})=\int_{S} \operatorname{ch}\left(\mathcal{E}^{\vee}\right) \operatorname{ch}(\mathcal{F}) \operatorname{Td}(T S) .
$$

In the case in which we have a couple of $D 7$ branes intersecting transversely over a curve $C$, the calculation of the Ext groups also simplifies [49]:

$$
\operatorname{Ext}^{i+1}\left(i_{*} \mathcal{E}, j_{*} \mathcal{F}\right)=H^{i}\left(S, F_{\mathcal{E}}^{\vee} \otimes F_{\mathcal{F}} \otimes \sqrt{K_{\mathcal{C}}}\right)
$$

for $i<2$, and 0 otherwise. If we are interested in purely computing indices, the result can again be expressed in terms of integrals of characteristic classes:

$$
\sum(-1)^{i} \operatorname{dim} \operatorname{Ext}^{i}\left(i_{*} \mathcal{E}, j_{*} \mathcal{F}\right)=\int_{C} \operatorname{ch}\left(F_{\mathcal{F}}^{\vee} \otimes F_{\mathcal{E}}\right)
$$

It is illuminating and useful to rewrite the formulas above as follows. Define the antisymmetric Dirac-Schwinger-Zwanziger (DSZ) product as:

$$
\left\langle\Gamma_{\mathcal{E}}, \Gamma_{\mathcal{F}}\right\rangle=\sum_{n=0}^{3} \int_{X}(-1)^{n} \Gamma_{\mathcal{E}}^{(2 n)} \wedge \Gamma_{\mathcal{F}}^{(6-2 n)},
$$

where $\Gamma^{(k)}$ denotes the part of the form $\Gamma$ of degree $k$. Then it is an easy exercise to check, given the index formulas above, that

$$
\sum(-1)^{i} \operatorname{dim} \operatorname{Ext}^{i}\left(i_{*} \mathcal{E}, j_{*} \mathcal{F}\right)=\left\langle\Gamma_{\mathcal{E}}, \Gamma_{\mathcal{F}}\right\rangle
$$

Notice that in our context the 6 -form part of $\Gamma$ plays no role, since its magnetic dual, the 0 -form, is always absent. We will thus often ignore the 6 -form part without further notice in any computation of chiral quantities.

As an illustration, the quiver for our example can now be reconstructed easily using (3.30) and the charges in (3.23):

$$
\begin{aligned}
\left\langle\Gamma_{\mathcal{E}_{1}}, \Gamma_{\mathcal{E}_{2}}\right\rangle & =\left\langle[S] \wedge\left(-1-\frac{3}{2} H\right),[S] \wedge(2+2 H)\right\rangle \\
& =\int_{S}\left(\left(-\frac{3}{2} H\right) \wedge 2[S]-2 H \wedge(-[S])\right) \\
& =6\left(\frac{3}{2}-1\right) \int_{S} H^{2} \\
& =3
\end{aligned}
$$

and similarly $\left\langle\Gamma_{\mathcal{E}_{1}}, \Gamma_{\mathcal{E}_{3}}\right\rangle=-3,\left\langle\Gamma_{\mathcal{E}_{2}}, \Gamma_{\mathcal{E}_{3}}\right\rangle=3$.

\subsection{Flavor $D 7$ branes}

Looking to the MSSM quiver in figure 4, we see that there are three basic flavor D7 branes we can consider, classified by to which fractional branes they couple to. In particular, in figure 4 we have a rank 6 stack coupling to $\mathcal{E}_{3}$ and $\mathcal{E}_{2}$ (but not $\mathcal{E}_{1}$ ), a rank 3 stack coupling 
only to $\mathcal{E}_{3}$ and $\mathcal{E}_{2}$, and finally a rank 0 stack (which we have not drawn), coupling only to $\mathcal{E}_{2}$ and $\mathcal{E}_{1}$. We will denote them respectively as $\mathcal{F}_{6}, \mathcal{F}_{3}$ and $\mathcal{F}_{0}$. Our task is to promote $\mathcal{F}_{3 i}$ to geometrical objects in the Calabi-Yau background.

We can obtain a first piece of information by imposing that the right bifundamentals exist between the gauge and flavor branes. Given that we are computing intersection numbers, we will only obtain in this way a restricted amount of information about $\Gamma_{\mathcal{F}_{i}}$. Let us make this explicit by parametrizing:

$$
\Gamma_{\mathcal{F}_{i}}=a_{i}\left[D_{H}\right]+b_{i}\left[D_{H}\right]^{2},
$$

where the only thing we need to know about $\left[D_{H}\right]$ is that $\left.\left[D_{H}\right]\right|_{\mathbb{P}^{2}}=H$. Looking to figure 4 , we have to impose that:

$$
\left\langle\Gamma_{\mathcal{E}_{1}}, \Gamma_{\mathcal{F}_{3}}\right\rangle=1
$$

which implies

$$
\left\langle[S] \wedge\left(-1-\frac{3}{2} H\right),\left[D_{H}\right] \wedge\left(a_{3}+b_{3}\left[D_{H}\right]\right)\right\rangle=\left(-\frac{3}{2} a_{3}+b_{3}\right) \int_{S} H^{2}=1 .
$$

Similarly, $\left\langle\Gamma_{\mathcal{E}_{2}}, \Gamma_{\mathcal{F}_{3}}\right\rangle=0$ implies:

$$
\left\langle[S] \wedge(2+2 H),\left[D_{H}\right] \wedge\left(a_{3}+b_{3}\left[D_{H}\right]\right)\right\rangle=2\left(a_{3}-b_{3}\right)=0 .
$$

These two equations together imply that:

$$
\Gamma_{\mathcal{F}_{3}}=\left[D_{H}\right] \wedge\left(-2-2\left[D_{H}\right]\right) .
$$

The third condition $\left\langle\Gamma_{\mathcal{E}_{3}}, \Gamma_{\mathcal{F}_{3}}\right\rangle=-1$ is now automatic, since $\sum_{i} \Gamma_{\mathcal{E}_{i}}=0$ (up to a 6 -form), and the DSZ product is linear.

We can proceed similarly for $\Gamma_{\mathcal{F}_{6}}$ and $\Gamma_{\mathcal{F}_{0}}$, obtaining:

$$
\begin{aligned}
& \Gamma_{\mathcal{F}_{6}}=\left[D_{H}\right] \wedge\left(1+\frac{3}{2}\left[D_{H}\right]\right) \\
& \Gamma_{\mathcal{F}_{0}}=\left[D_{H}\right] \wedge\left(1+\frac{1}{2}\left[D_{H}\right]\right)
\end{aligned}
$$

We now lift this local information to global information. As an illustration, we will embed our local system into the compact Calabi-Yau in section 2.2, namely an elliptic fibration over $\mathbb{P}^{2}$. In doing this, global tadpoles with charge in the non-compact divisors will not be canceled, but we will ignore this effect momentarily, coming back to it later. Recall from section 2.2 that $D_{6}$ described our local $\mathbb{P}^{2}$. It is also easy to see that $\left.D_{1}\right|_{D_{6}}=H$, for example by computing the triple intersection number $D_{1} \cdot D_{1} \cdot D_{6}=1$ in the CalabiYau. In order to emphasize the fibration structure of the threefold, we will relabel our coordinates

$$
(s, t, u, x, y, z) \equiv\left(x_{1}, x_{2}, x_{3}, x_{4}, x_{5}, x_{6}\right) .
$$


From table 1 , we see that $(s, t, u)$ are the coordinates of the $\mathbb{P}^{2}$, located at $z=0 \cap X_{3}$. Similarly, the elliptic curve is the hypersurface in the $\mathbb{P}^{2,3,1}$ described by $(x, y, z)$.

A generic $D 7$ brane $\mathcal{F}$ on our global embedding can be described by:

$$
\Gamma_{\mathcal{F}}=\left[a D_{s}+b D_{z}\right] \wedge\left(r+c D_{s}+d D_{z}\right) \wedge\left(1-\frac{1}{2}\left(a D_{s}+b D_{z}\right)\right)
$$

with $a, b, c, d, r \in \mathbb{Z}$, and the last term comes from expanding $\sqrt{\operatorname{Td}(T D) / \operatorname{Td}(N D)}$ (here $\left.D \equiv a D_{s}+b D_{z}\right)$. We have ignored 6-form terms, as usual. Notice that the divisor $a D_{s}+b D_{z}$ will in general have more two-forms than those induced from the ambient space, so we could add further 4 -form terms (an example is $D_{s}$ itself). Nevertheless, since the DSZ product is taken in $X$, it will not see these, so we have set them to 0 . We want the flavor branes to look like ordinary $D 7$ branes away from the singularity, so we impose $r=a=1:^{11}$

$$
\Gamma_{\mathcal{F}}=\left[D_{s}+b D_{z}\right] \wedge\left(1+\left(c-\frac{1}{2}\right) D_{s}+\left(d-\frac{b}{2}\right) D_{z}\right) .
$$

Using the restrictions $\left.D_{s}\right|_{D_{z}}=H,\left.D_{z}\right|_{D_{z}}=-3 H$ :

$$
\left.\Gamma_{\mathcal{F}}\right|_{D_{z}}=(1-3 b) H \wedge\left(1+\left(c-3 d-\frac{1-3 b}{2}\right) H\right) .
$$

From here it is easy to read the global information given by the quiver. We find that:

$$
\begin{aligned}
& \Gamma_{\mathcal{F}_{6}}=\left[D_{s}\right] \wedge\left(1+\left(3 d_{6}+2\right)\left[D_{s}\right]+d_{6}\left[D_{z}\right]\right) \wedge \sqrt{\frac{T D_{s}}{N D_{s}}} \\
& \Gamma_{\mathcal{F}_{3}}=\left[D_{s}+D_{z}\right] \wedge\left(1+3 d_{3}\left[D_{s}\right]+d_{3}\left[D_{z}\right]\right) \wedge \sqrt{\frac{T\left(D_{s}+D_{z}\right)}{N\left(D_{s}+D_{z}\right)}} \\
& \Gamma_{\mathcal{F}_{0}}=\left[D_{s}\right] \wedge\left(1+\left(3 d_{0}+1\right)\left[D_{s}\right]+d_{0}\left[D_{z}\right]\right) \wedge \sqrt{\frac{T D_{s}}{N D_{s}}}
\end{aligned}
$$

Notice that there is certain ambiguity, and with what we have said so far one can only fix $c-3 d$, but not $c$ and $d$ individually. We have reflected this ambiguity in the unknown coefficients $d_{i} \in \mathbb{Z}$.

\subsection{D5-brane tadpole}

Our original motivation for introducing flavor branes was that D5 brane charge was not canceled with the desired assignment of ranks for the gauge groups, and this induced a tadpole. As a consistency check, let us now verify that the local tadpole cancels once we introduce the flavor branes.

\footnotetext{
${ }^{11}$ Since the cycle $D_{z}$ is the one wrapping the contracting four-cycle, away from the singularity the $D 7$ wraps a divisor of the form $r a D_{s}$, which in the local geometry $\mathbb{C}[x, y, z] / \mathbb{Z}_{3}$ is described by a polynomial in $x, y, z$ of degree $r a$. An ordinary flavor brane is described by a linear polynomial $(x=0$, say), so we set $r a=1$. Since both $r$ and $a$ are integers, and the relative sign is conventional, we are free to choose $r=a=1$.
} 
We denote the curves Poincare dual to $D_{s}$ and $D_{z}$ by $\ell_{s}$ and $\ell_{z}$, respectively. These curves are defined in homology by $\ell_{s} \cdot D_{s}=\ell_{z} \cdot D_{z}=1$ and $\ell_{s} \cdot D_{z}=\ell_{z} \cdot D_{s}=0$. By comparing with curves of the form $D_{i} \cdot D_{j}$, we find that

$$
\begin{aligned}
& \ell_{z}=D_{s} \cdot D_{s} \\
& \ell_{s}=D_{s} \cdot\left(3 D_{s}+D_{z}\right) .
\end{aligned}
$$

$D 5$ tadpole cancellation requires that all $D 5$ brane charge is supported on a 2-cycle that does not intersect any compact 4-cycle. Since the compact 4-cycle in the non-compact geometry is $D_{z}$, we need all of our $D 5$ charge to be supported on $\ell_{s}$.

Recall from (3.45) that a general flavor $D 7$ brane $\mathcal{F}$ has a charge vector:

$$
\Gamma_{\mathcal{F}}=\left[D_{s}+b D_{z}\right] \wedge\left(1+\left(c-\frac{1}{2}\right) D_{s}+\left(d-\frac{b}{2}\right) D_{z}\right) .
$$

The $D 5$ charge is given by the 4 -form part of (3.45):

$$
\begin{aligned}
\Gamma_{\mathcal{F}}^{(D 5)} & =\left(c-\frac{1}{2}\right) D_{s} \cdot D_{s}+\left[\left(c-\frac{1}{2}\right) b+\left(d-\frac{b}{2}\right)\right] D_{s} \cdot D_{z}+b\left(d-\frac{b}{2}\right) D_{z} \cdot D_{z} \\
& =\left[d+b\left(c-3 d-1+\frac{3 b}{2}\right)\right] \ell_{s}+(1-3 b)\left(c-3 d-\frac{1-3 b}{2}\right) \ell_{z}
\end{aligned}
$$

where in the second line we have used (3.48), and the fact that $D_{z} \cdot D_{z}=9 \ell_{z}-3 \ell_{s}$. As one may have expected, the $\ell_{z}$ charge is precisely the 4-form term in the restriction of the flavor brane charge to $D_{z}$ (recall (3.46)).

Using this last observation, and (3.40)-(3.42) it is easy to compute the contribution of the flavor branes to the compact $D 5$ tadpole; in our case:

$$
\begin{aligned}
\Gamma_{\mathcal{F}}^{(D 5)} & =6 \Gamma_{\mathcal{F}_{6}}^{(D 5)}+3 \Gamma_{\mathcal{F}_{3}}^{(D 5)} \\
& =\left(6 d_{6}+3 d_{3}+\frac{3}{2}\right) \ell_{s}+3 \ell_{z}
\end{aligned}
$$

The fractional branes can be dealt with similarly. A general fractional brane can be written as:

$$
\Gamma_{\mathcal{E}}=D_{z} \wedge\left(a+b D_{s}\right)
$$

The $D 5$ brane charge is thus given by $b D_{s} \cdot D_{z}=b\left(\ell_{s}-3 \ell_{z}\right)$. Using the charges given in (3.23), we then find that: ${ }^{12}$

$$
\Gamma_{\mathcal{E}}^{(D 5)}=3 \Gamma_{\mathcal{E}_{3}}^{(D 5)}+2 \Gamma_{\mathcal{E}_{2}}^{(D 5)}+\Gamma_{\mathcal{E}_{1}}^{(D 5)}=\ell_{s}-3 \ell_{z} .
$$

And as we see, this cancels the $\ell_{z}$ part of (3.50), leaving:

$$
\Gamma_{\mathcal{F}}^{(D 5)}+\Gamma_{\mathcal{E}}^{(D 5)}=\left(6 d_{6}+3 d_{3}+\frac{5}{2}\right) \ell_{s} .
$$

\footnotetext{
${ }^{12}$ There is a slight clash of notation here. Notice that $[S]$ in $(3.23)$ refers to $D_{z}$, not $D_{s}$.
} 
Let us point out that global tadpole cancellation requires all D5 charge to vanish, once we embed the local geometry into a compact model. This is clearly not possible in (3.53), since $d_{i} \in \mathbb{Z}$, but this is clearly a shortcoming of this particular (oversimplified) embedding, which, as we will see, has problems even at the level of canceling $D 7$ brane charge. In general cases where the $D 7$ sector admits a consistent embedding we expect the equation analogous to (3.53) to admit solutions.

\section{Type IIB orientifolds}

Next we turn to the study of various orientifold involutions, anticipating the D-brane/open string sector required for the gauge theory/matter, and how to up-lift this to F-theory. We will mostly follow the generalization of Sen's original work connecting type IIB orientifolds and F-theory [54-56], as can be found in [57-59].

\subsection{Generalities}

With the inclusion of the open string sector, and the associated D-brane charge, we need to construct the orientifold model from the Calabi-Yau manifold in which the local geometry was embedded, in order to satisfy the various tadpole conditions. The choice of the orientifold involution $\sigma$ has to be made such that the fixed point set left invariant under $\sigma$ gives a divisor which when wrapped by the orientifold plane leads to tadpole cancellation. For the D7-brane charge this corresponds to

$$
8 D_{O 7^{-}}=D_{D 7}
$$

in conventions of the double cover, i.e., counting each $D 7$ and its image separately.

One possible type of orientifold involution that will be of particular interest to us is a permutation symmetry acting on the coordinates of the ambient space,

$$
\sigma:\left(x_{0}, \ldots, x_{n}\right) \leftrightarrow\left(x_{\sigma(0)}, \ldots, x_{\sigma(n)}\right),
$$

where $x_{\sigma(i)}$ refers to the coordinate which $x_{i}$ is mapped to under the orientifold involution $\sigma$. The points in the fixed locus are defined by:

$$
\left(x_{0}, \ldots, x_{n}\right)=\mathfrak{g}\left[\left(x_{\sigma(0)}, \ldots, x_{\sigma(n)}\right)\right],
$$

where $\mathfrak{g}$ is a gauge transformation of the underlying gauged sigma model.

The reader may wonder whether (4.2) is a well defined action on the toric ambient space, since it is not obvious that it commutes with the gauge action. That this is the case can be argued in general as follows. Start from the fact that the generators of the Mori cone give a basis for the U(1) generators of the GLSM, with charges of the divisors given by the intersection numbers between the divisor and the Mori cone generator. Now assume that we have a permutation involution $\sigma$, (4.2), of the toric fan. In terms of the GLSM, this induces a $\mathbb{Z}_{2}$ permutation of the columns. We now argue that this permutation of the columns of the GLSM can be undone by a permutation of the rows. 
Calling $\ell_{i}$ the Mori cone generators, and $D_{j}$ the toric divisors, one has that $\ell_{i} \cdot D_{j}=$ $\sigma\left(\ell_{i}\right) \cdot \sigma\left(D_{j}\right)$. Since this is an involution of the fan we have that $\sigma\left(\ell_{i}\right)=\ell_{k}$, i.e. one of the original charges. Another way of saying this is that the permutation involution is a permutation of the generators of the Mori cone, if we choose the involution to be an involution of the fan. Thus the permutation of the columns can be undone by (i.e., it is equivalent to, since we are in $\mathbb{Z}_{2}$ ) a permutation of the rows.

From here it is easy to see that the permutation orbifold is well defined. We need to show that, for any gauge transformation $\mathfrak{g}_{1}$ of the GLSM, $\mathfrak{g}_{2} \equiv \sigma \mathfrak{g}_{1} \sigma$ is another gauge transformation of the GLSM. Looking to the coordinates, it is easy to convince oneself that $\sigma \mathfrak{g}_{1} \sigma$ induces the action on the Mori cone given above, and thus an action on the charge vector which amounts to a relabeling.

Given any $\mathbb{Z}_{2}$ action on the coordinates of the ambient space, (4.2) or otherwise, we can construct the quotient space by constructing the set of coordinates invariant under the orientifold action, and imposing any relations that follow tautologically from the definition of the invariant coordinates in terms of the original coordinates. There will be no constraints between the invariant coordinates in our examples below, but they can appear in general, as the following example shows. Consider the $\mathbb{C}^{2} / \mathbb{Z}_{2}$ orbifold, with orbifold action on the $x_{1}, x_{2}$ coordinates of $\mathbb{C}$ given by:

$$
\left(x_{1}, x_{2}\right) \rightarrow\left(-x_{1},-x_{2}\right)
$$

Invariant coordinates are given by $a=x_{1}^{2}, b=x_{2}^{2}, c=x_{1} x_{2}$. It is easy to convince oneself that any invariant polynomial can be constructed out of these coordinates, so $a, b, c$ generate the ring of functions on the quotient. Due to their definition, the constraint between the coordinates is given by:

$$
a b=c^{2}
$$

We thus reproduce the well-known fact that $\mathbb{C}^{2} / \mathbb{Z}_{2}$ is alternatively described as the hypersurface $(4.5)$ in $\mathbb{C}^{3}$.

\subsection{Sign orientifolds: $M_{d P_{0}}$}

In section 3 we have described in large volume terms the flavor and color branes in our local model. As a result of this analysis, we found that the total $D 7$ brane charge in the embedding of $\mathbb{C}^{3} / \mathbb{Z}_{3}$ into $M_{d P_{0}}$ is given by (recall (3.47)):

$$
Q_{D 7}=9 D_{s}+3 D_{z} \sim D_{y}
$$

Notice that this charge is concentrated on a divisor which does not intersect $D_{z}$, consistent with local tadpole cancellation. In order to cancel the resulting global tadpole while keeping a supersymmetric model, we need to introduce a $O 7^{-}$orientifold. The $M_{d P_{0}}$ embedding is too simple, so cannot introduce the orientifold without also perturbing the local model, but it serves as a good stepping stone to the more realistic model in section 4.3. 
In view of the relations (4.1) and (4.6), we cancel the tadpoles by wrapping an orientifold on $D_{y}$, and adding extra branes on top of $D_{y}$. This is easy to achieve by quotienting the space by the involution:

$$
(s, t, u, x, y, z) \rightarrow(s, t, u, x,-y, z) .
$$

This involution also acts on the equation defining the Calabi-Yau hypersurface, and we should make sure that the involution and the Calabi-Yau are compatible. The most general Calabi-Yau hypersurface in the ambient space is given by (ignoring the $\mathbb{Z}_{2}$ action for a moment):

$$
y^{2}+a_{1} x y z+a_{3} y z^{3}=x^{3}+a_{2} x^{2} z^{2}+a_{4} x z^{4}+a_{6} z^{6},
$$

where $a_{n}$ is a homogeneous polynomial of order $3 n$ on the $s, t, u$ variables. If we now impose invariance under (4.7), the fixed locus is indeed at $D_{y}$. It is easy to see that this involution is compatible with the equation (4.8) defining the Calabi-Yau hypersurface if we set $a_{1}=a_{3}=0$. The gauge invariance of the background forces us to consider also the following $\mathbb{Z}_{2}$ actions:

$$
\begin{aligned}
(s, t, u, x, y, z) & \rightarrow(s, t, u, x, y,-z) \\
(s, t, u, x, y, z) & \rightarrow(-s,-t,-u, x, y, z) .
\end{aligned}
$$

The second involution has no fixed points, since $\{s=t=u=0\}$ is in the Stanley-Reisner ideal (2.5), but the first involution has fixed points at $\{z=0\} \sim D_{z}$. In order to cancel the charge coming from this component of the orientifold, according to (4.1), we need to wrap extra $D 7$ branes on $D_{z}$, the cycle supporting the gauge sector. This will render a consistent model, but not the one that we wanted to embed.

\subsection{Permutation orientifolds: $M_{\left(d P_{0}\right)^{3}}$}

With hindsight, the fact that the model in the previous section required important modifications to the open string sector is not too surprising. The orientifold that we chose leaves all divisors classes invariant, and thus typically will project down some unitary gauge factors to symplectic or orthogonal subgroups, in addition to identifying nodes in the quiver. What we want is an involution that exchanges the gauge sector with a copy somewhere else in the Calabi-Yau, without the orientifold intersecting the gauge sector.

In this section we discuss a simple extension of the model of the previous section that achieves this. In fact, we have already encountered a suitable geometry: it is the $M_{\left(d P_{0}\right)^{3}}$ geometry we discussed in section 2.4. ${ }^{13}$ We show the action of the involution on the local geometry in figure 8 . The induced $\mathbb{Z}_{2}$ action on $M_{\left(d P_{0}\right)^{3}}$ is given by:

$$
\left(x_{0}, x_{1}, \ldots, x_{10}, x_{11}\right) \leftrightarrow\left(x_{0}, x_{1}, x_{2}, x_{4}, x_{3}, x_{10}, x_{9}, x_{7}, x_{11}, x_{6}, x_{5}, x_{8}\right) .
$$

\footnotetext{
${ }^{13}$ The hyperconifold in section 2.3 also has an involution of the local geometry. Unfortunately this involution does not extend to the global embedding $M_{Y^{3,0}}$ that we chose. $M_{\left(d P_{0}\right)^{3}}$ also has the advantage of having a divisor mapped to itself under the orientifold involution, and which could thus be interesting from the point of view of generating non-perturbative superpotentials.
} 


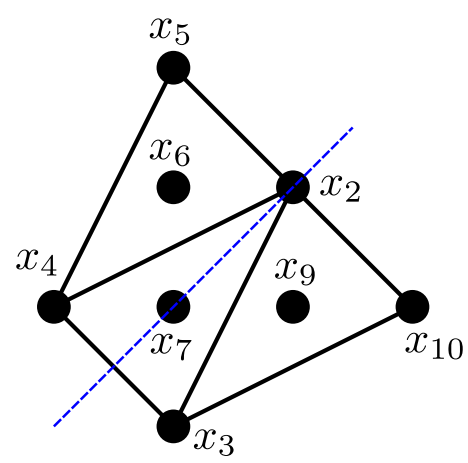

Figure 8. Local geometry for the model in this section. The orientifold involution acts by reflection on the dashed blue line. We have partially resolved the singularity into three separated $\mathbb{C}^{3} / \mathbb{Z}_{3}$ singularities. We have also indicated the position of the coplanar divisors in (2.31).

According to the discussion in section 4.1, this action can equivalently be seen as an action on the Mori cone generators (2.35):

$$
\left(\hat{\ell}_{3}, \hat{\ell}_{5}, \hat{\ell}_{6}, \hat{\ell}_{8}\right) \leftrightarrow\left(\hat{\ell}_{9}, \hat{\ell}_{7}, \hat{\ell}_{2}, \hat{\ell}_{4}\right)
$$

with $\hat{\ell}_{1}$ fixed.

Identifying the monomials invariant under involution (4.11) we obtain the quotient map

$$
\begin{aligned}
\left(x_{0}, \cdots, x_{11}\right) \rightarrow & \left(z_{0}, \cdots, z_{8}\right) \\
& =\left(x_{0}, x_{1}, x_{2}, x_{3} x_{4}, x_{5} x_{10}, x_{6} x_{9}, x_{8} x_{11}, x_{7}, x_{3} x_{9} x_{10}^{2} x_{11}+x_{4} x_{5}^{2} x_{6} x_{8}\right) .
\end{aligned}
$$

Notice in particular that the contracting $d P_{0}$ on which we want to put our gauge sector is now given by $z_{5}=0$.

The points in the fixed locus satisfy:

$$
\left(x_{0}, x_{1}, \ldots, x_{10}, x_{11}\right)=\mathfrak{g}\left[\left(x_{0}, x_{1}, x_{2}, x_{4}, x_{3}, x_{10}, x_{9}, x_{7}, x_{11}, x_{6}, x_{5}, x_{8}\right)\right],
$$

where $\mathfrak{g}$ is a gauge transformation of the GLSM $(2.32)$, in other words an element of $\left(\mathbb{C}^{*}\right)^{8}$. Condition (4.14) can be seen to be equivalent to:

$$
x_{8} x_{5}^{2} x_{4} x_{6}=x_{3} x_{10}^{2} x_{9} x_{11},
$$

and thus the fixed locus is in the divisor class

$$
D_{O 7}=-D_{2}+D_{3}+D_{4} .
$$

Notice that, as one may guess by looking to figure 8 , the orientifold locus does not intersect the cycle on which we are wrapping our branes, even in the ambient space. Take for example the $d P_{0}$ coming from the $x_{6}=0$ divisor. If this cycle intersected the orientifold locus, from (4.15) this would imply that $x_{3} x_{10}^{2} x_{9} x_{11}=0$, but notice that all of $x_{6} x_{3}, x_{6} x_{10}$, 
$x_{6} x_{9}$ and $x_{6} x_{11}$ are in the Stanley-Reisner ideal (2.34), and thus $x_{3} x_{10}^{2} x_{9} x_{11} \neq 0$ when $x_{6}=0$.

One interesting subtlety that appears in this example, and not in the examples studied in [57-59], is the following. Notice that we constructed 9 invariant coordinates, but the dimension of the Mori cone before the involution was 8 . We also constructed the action on the Mori cone generators in (4.12), and we saw that it permutes 8 generators in pairs. It is thus a little bit puzzling how one would get the $9-4=5$ Mori cone generators that one would need in order to construct the ambient fourfold where the base lives as a hypersurface. Furthermore, it is easy to convince oneself that the $z_{i}$ coordinates do not satisfy any identity.

This is resolved as follows. Notice that because the Mori cone is not simplicial, in order to generate it we needed 9 effective curves, rather than 8 than dimension counting may suggest. There is a linear relation between the Mori cone generators in (2.35) (this is easy to verify for instance by computing the rank of (2.35) seen as a matrix), but interestingly, once we quotient by the orientifold action, the linear relation no longer holds, and we generate a new Mori cone generator, for a total of 5, as one expects. The ambient space $\mathcal{A}_{\left(d P_{0}\right)^{3}} / \mathbb{Z}_{2}$ of the quotient is then described by the set of Mori cone generators invariant under (4.12):

$$
\begin{array}{r|rrrrrrrrr} 
& z_{0} & z_{1} & z_{2} & z_{3} & z_{4} & z_{5} & z_{6} & z_{7} & z_{8} \\
\hline \hat{\ell}_{1} \sim \check{\ell}_{0} & 0 & 0 & 1 & 2 & 0 & 0 & 0 & -3 & 1 \\
\hat{\ell}_{2} \sim \hat{\ell}_{6} \sim \check{\ell}_{1} & 0 & 0 & -1 & -1 & 0 & 1 & 0 & 1 & 0 \\
\hat{\ell}_{3} \sim \hat{\ell}_{9} \sim \check{\ell}_{2} & 1 & 0 & 0 & 0 & -2 & 1 & 3 & 0 & 0 \\
\hat{\ell}_{4} \sim \hat{\ell}_{8} \sim \check{\ell}_{3} & 0 & -1 & 1 & 1 & 0 & -3 & 2 & 0 & 0 \\
\hat{\ell}_{5} \sim \hat{\ell}_{7} \sim \check{\ell}_{4} & 0 & 1 & 0 & 0 & 1 & 0 & -2 & 0 & 0
\end{array}
$$

Here we have denoted the Mori cone generators by $\check{\ell}_{i}$, and we have also indicated where they come from in the double quotient. The resulting space is a toric variety, given by the polytope:

$$
\begin{array}{rrrrrrrrrr}
z_{0} & z_{1} & z_{2} & z_{3} & z_{4} & z_{5} & z_{6} & z_{7} & z_{8} \\
\hline 1 & 0 & 0 & 0 & 6 & 2 & 3 & -2 & -6 \\
0 & 1 & 0 & 0 & 9 & 3 & 5 & -3 & -9 \\
0 & 0 & 1 & 0 & 2 & 1 & 1 & 0 & -1 \\
0 & 0 & 0 & 1 & 2 & 1 & 1 & 0 & -2
\end{array}
$$

and Stanley-Reisner ideal:

$$
\begin{array}{r}
\operatorname{SR}\left(\mathcal{A}_{\left(d P_{0}\right)^{3} / \mathbb{Z}_{2}}\right)=\left\langle z_{1} z_{4}, z_{6} z_{7}, z_{6} z_{8}, z_{4} z_{8}, z_{5} z_{8}, z_{5} z_{7}, z_{4} z_{7}, z_{0} z_{1} z_{5},\right. \\
\left.z_{0} z_{1} z_{7}, z_{0} z_{5} z_{6}, z_{2} z_{3} z_{6}, z_{2} z_{3} z_{8}, z_{2} z_{3} z_{4}, z_{0} z_{1} z_{2} z_{3}\right\rangle .
\end{array}
$$

After the involution (4.12), the original Calabi-Yau equation $P\left(x_{i}\right)=0$ with degrees

$$
\operatorname{deg}(P)=(0,0,3,0,0,0,0,0,3)
$$


(see the $K$ column in (2.35)) becomes an equation $\check{P}\left(z_{i}\right)=0$ of degree

$$
\operatorname{deg}(\check{P})=(0,0,3,0,0) .
$$

On the other hand, the degrees of the anti-canonical class of $\mathcal{A}_{\left(d P_{0}\right)^{3}} / \mathbb{Z}_{2}$ are given by:

$$
\operatorname{deg}\left(\bar{K}_{\mathcal{A}_{\left(d P_{0}\right)^{3}} / \mathbb{Z}_{2}}\right)=(1,0,3,0,0),
$$

and from here we easily deduce the anti-canonical class of the hypersurface $\mathcal{B}_{3}=\left\{\check{P}\left(z_{i}\right)=0\right\}$ :

$$
\operatorname{deg}\left(\bar{K}_{\mathcal{B}_{3}}\right)=\operatorname{deg}\left(\bar{K}_{\mathcal{A}_{\left(d P_{0}\right)^{3}} / \mathbb{Z}_{2}}\right)-\operatorname{deg}(\check{P})=(1,0,0,0,0) .
$$

We will verify in section 5.1 that this agrees beautifully with what one would expect from Sen's limit, similarly to the discussion in [58].

Rather than adding the flavor branes directly in the type IIB orientifold setting we will find it more convenient to lift the above orientifold picture to F-theory, to which we turn next.

\section{$5 \quad$ Hybrid embeddings}

Let us present a systematic method for embedding the previous configurations in F-theory. Although F-theory adequately captures non-perturbative $g_{s}$ effects, naively it is ill-suited for describing branes at singularities, which require non-perturbative $\alpha^{\prime}$ effects for a full description. (Alternatively, if we want to describe fractional branes at large volume we have to deal with anti- $D 7$ branes in F-theory.)

The basic idea we use for overcoming this obstruction is the following: notice that by local tadpole cancellation, the total $D 7$-brane charge of the local configuration wraps a cycle that does not intersect the collapsing cycle. Thus, at the level of cohomology (what F-theory describes most naturally), the contracting cycle is generically far away from the discriminant! From the point of view of F-theory, our quiver configurations are then described by ordinary Calabi-Yau 4-fold compactifications at a very non-generic point in their moduli space, where the discriminant intersects a singular point in the geometry. This observation, while simple, is clearly very general. Let us illustrate how it works for our working example $M_{\left(d P_{0}\right)^{3}}$.

\section{$5.1 M_{\left(d P_{0}\right)^{3}}$}

We follow the techniques introduced in [57-59] to uplift IIB configurations to F-theory. One starts with the threefold base $\mathcal{B}_{3}$ of the fourfold obtained by taking the $\mathbb{Z}_{2}$ quotient of $M_{\left(d P_{0}\right)^{3}}$, as constructed in section 4.3. In general, one can present a Calabi-Yau fourfold elliptically fibered over a base $\mathcal{B}_{3}$ using a Weierstrass form: ${ }^{14}$

$$
y^{2}=x^{3}+f x z^{4}+g z^{6},
$$

\footnotetext{
${ }^{14} \mathrm{As}$ it is conventional when writing elliptic fibrations, we denote the coordinates on the fiber by $x, y, z$. We hope the reader will not get confused by the unrelated elliptic fibration and corresponding $x, y, z$ coordinates studied in section 3.2 .
} 
with $f$ a section of $K_{\mathcal{B}_{3}}^{-4}$ and $g$ a section of $K_{\mathcal{B}_{3}}^{-6}$. Taking Sen's limit, one has that

$$
f \sim-3 h^{2}+\ldots
$$

with $h=0$ the orientifold locus. In 4.3 we found that the orientifold locus in the Calabi-Yau threefold is located at

$$
\sqrt{h}=x_{3} x_{9} x_{10}^{2} x_{11}-x_{4} x_{5}^{2} x_{6} x_{8}=0
$$

with the square root encoding the fact that the Calabi-Yau threefold is the double cover of $\mathcal{B}_{3}$, where $h$ is most naturally defined. We thus have that:

$$
\begin{aligned}
h & =\left(x_{3} x_{9} x_{10}^{2} x_{11}-x_{4} x_{5}^{2} x_{6} x_{8}\right)^{2} \\
& =\left(x_{3} x_{9} x_{10}^{2} x_{11}+x_{4} x_{5}^{2} x_{6} x_{8}\right)^{2}-4 x_{3} x_{4} x_{6} x_{9}\left(x_{5} x_{10}\right)^{2} x_{8} x_{11} \\
& =z_{8}^{2}-4 z_{3} z_{4}^{2} z_{5} z_{6},
\end{aligned}
$$

which has degree $(2,0,0,0,0)=\operatorname{deg}\left(\bar{K}_{\mathcal{B}_{3}}^{2}\right)$. Taking into account (5.2) and (4.23) this gives the expected degree $\operatorname{deg}\left(\bar{K}_{\mathcal{B}_{3}}^{4}\right)$ for $f$.

In addition to having constructed the Calabi-Yau fourfold itself, we want to go to a point in moduli space where the flavor and gauge $D 7$ branes have the right structure close to the singularity. From the local analysis in section 3.2 (see in particular eqs. (3.47)) we want the discriminant to degenerate as a rank 6 stack of branes wrapping the non-compact divisor intersecting the collapsing $d P_{0}$ on its hyperplane class (this stack will give rise to $\mathcal{F}_{6}$ ), and a rank 3 stack wrapping the same non-compact divisor plus the collapsing $d P_{0}$ itself (this stack will give rise to $\mathcal{F}_{3}$ ).

It is not hard to see that there are indeed locations in the moduli space of the elliptic fibration such that the discriminant has this structure. In order to explicitly obtain these loci it is easiest to work with the elliptic fibration in its Tate form [60]:

$$
y^{2}+a_{1} x y z+a_{3} y z^{3}=x^{3}+a_{2} x^{2} z^{2}+a_{4} x z^{4}+a_{6} z^{6},
$$

where the $a_{i}$ are sections of $K_{\mathcal{B}_{3}}^{-i}$. In order to have a $\mathrm{U}(3)$ stack on $z_{5}=0$ (the locus of the contracting cycle, recall the map (4.13)), we impose the following degrees of vanishing [61]:

$$
\begin{array}{ll}
\operatorname{deg}\left(a_{1}\right)=0 ; \quad \operatorname{deg}\left(a_{2}\right)=1 ; \quad \operatorname{deg}\left(a_{3}\right)=1 \\
\operatorname{deg}\left(a_{4}\right)=2 ; \quad \operatorname{deg}\left(a_{6}\right)=3,
\end{array}
$$

with the notation meaning that close to the $z_{5}=0$ locus, $a_{i}$ vanishes as $z_{5}^{\operatorname{deg}\left(a_{i}\right)}(\ldots)$, with the quantity in parenthesis generically non-vanishing. We find the following space of 
solutions:

$$
\begin{aligned}
& a_{1}=c_{11} z_{2} z_{4}^{2} z_{5} z_{6}+c_{12} z_{8} \\
& a_{2}=z_{5}\left(c_{21} z_{2}^{2} z_{4}^{4} z_{5} z_{6}^{2}+c_{22} z_{2} z_{4}^{2} z_{6} z_{8}+c_{23} z_{3} z_{4}^{2} z_{6}\right) \\
& a_{3}=z_{5}\left(c_{31} z_{2}^{3} z_{4}^{6} z_{5}^{2} z_{6}^{3}+c_{32} z_{2} z_{4}^{2} z_{6} z_{8}^{2}+c_{33} z_{3} z_{4}^{2} z_{6} z_{8}\right. \\
& \left.+c_{34} z_{2} z_{3} z_{4}^{4} z_{5} z_{6}^{2}+c_{35} z_{2}^{2} z_{4}^{4} z_{5} z_{6}^{2} z_{8}\right) \\
& a_{4}=z_{5}^{2}\left(c_{41} z_{2}^{4} z_{4}^{8} z_{5}^{2} z_{6}^{4}+c_{42} z_{2}^{2} z_{4}^{4} z_{6}^{2} z_{8}^{2}+c_{43} z_{3}^{2} z_{4}^{4} z_{6}^{2}\right. \\
& \left.+c_{44} z_{2} z_{3} z_{4}^{4} z_{6}^{2} z_{8}+c_{45} z_{2}^{3} z_{4}^{6} z_{5} z_{6}^{3} z_{8}+c_{46} z_{2}^{2} z_{3} z_{4}^{6} z_{5} z_{6}^{3}\right) \\
& a_{6}=z_{5}^{3}\left(c_{61} z_{2}^{6} z_{4}^{12} z_{5}^{3} z_{6}^{6}+c_{62} z_{2}^{3} z_{4}^{6} z_{6}^{3} z_{8}^{3}+c_{63} z_{3}^{3} z_{4}^{6} z_{6}^{3}\right. \\
& +c_{64} z_{2}^{2} z_{3} z_{4}^{6} z_{6}^{3} z_{8}^{2}+c_{65} z_{2} z_{3}^{2} z_{4}^{6} z_{6}^{3} z_{8}+c_{66} z_{2}^{4} z_{4}^{8} z_{5} z_{6}^{4} z_{8}^{2} \\
& +c_{67} z_{2}^{3} z_{3} z_{4}^{8} z_{5} z_{6}^{4} z_{8}+c_{68} z_{2}^{2} z_{3}^{2} z_{4}^{8} z_{5} z_{6}^{4}+c_{69} z_{2}^{5} z_{4}^{10} z_{5}^{2} z_{6}^{5} z_{8} \\
& \left.+c_{6,10} z_{2}^{4} z_{3} z_{4}^{10} z_{5}^{2} z_{6}^{5}\right) \text {, }
\end{aligned}
$$

with the $c_{i j}$ arbitrary coefficients parametrizing the complex structure moduli space. It is a straightforward calculation to verify that the discriminant restricted to $z_{5}=0$ has the local form:

$$
\left.\frac{\Delta}{z_{5}^{3}}\right|_{z_{5}=0}=z_{4}^{6} z_{6}^{3} f\left(z_{0}, z_{1}, z_{2}, z_{3}, z_{7}, z_{8}\right) .
$$

With $f$ a section of $\mathcal{O}(3)$ (here, and it what follows, the line bundles are over $\mathbb{P}^{2}=\left\{z_{5}=0\right\}$ ). In order to see this, notice that, when restricted to $z_{5}=0$, the divisors $D_{i}=\left[\left\{z_{i}=0\right\}\right]$ become:

$$
\left.\left(D_{0}, D_{1}, D_{2}, D_{3}, D_{4}, D_{5}, D_{6}, D_{7}, D_{8}\right)\right|_{z_{5}=0}=(\mathcal{O}, \mathcal{O}, \mathcal{O}(1), \mathcal{O}(1), \mathcal{O}(1), \mathcal{O}(-3), \mathcal{O}, \mathcal{O}, \mathcal{O})
$$

Notice in particular that (4.23) and (4.17), imply that $\bar{K}_{\mathcal{B}_{3}}=D_{8}$, and thus $\left.\bar{K}_{\mathcal{B}_{3}}\right|_{z_{5}=0}=\mathcal{O}$, or in other words the geometry is locally Calabi-Yau, as we expected. Since $\Delta$ is a section of $\bar{K}_{\mathcal{B}_{3}}^{12}$, and thus trivial when restricted to $z_{5}=0$, and we have split off a factor of $z_{5}^{3} z_{4}^{6} z_{6}^{3}$, which is locally a section of $\mathcal{O}(3 \cdot(-3)+6 \cdot 0+3 \cdot 1)=\mathcal{O}(-3)$, we conclude that $f$ is a section of $\mathcal{O}(3)$, or in other words a cubic polynomial on the local coordinates of the contracting $\mathbb{P}^{2}$.

It is natural to associate the $\mathrm{U}(6)$ flavor stack with $z_{4}=0$. One may be tempted to interpret the $z_{6}^{3}$ factor in (5.8) as the non-compact part of the $\mathrm{U}(3)$ stack. This is not correct, though: notice from (5.9) that $D_{6}$ becomes trivial when restricted to $z_{5}=0$, so $z_{6}$ is effectively a non-zero constant. Another way of seeing this is by recalling that the hypersurface defining $\mathcal{B}_{3}$ is in the same class as $z_{0}^{3}=0$, and $z_{0} z_{5} z_{6}$ is in the Stanley-Reisner ideal (4.19) of $\mathcal{A}_{\left(d P_{0}\right)^{3} / \mathbb{Z}_{2}}$.

The non-compact part of the $\mathrm{U}(3)$ stack must then come from $f$, and so we learn that generically the $\mathrm{U}(3)$ part of the flavor symmetry is broken in this embedding, since $f$ does not generically vanish to cubic order. This could be desirable for model building purposes, but one can also tune coefficients to locally recover the symmetry. One way of achieving this is to impose the vanishing degrees (5.6) for the coordinate $z_{2}$ (so, in particular, the 
discriminant behaves as $\left.\Delta=z_{2}^{3}(\ldots)\right)$. In this way we have a $\mathrm{U}(3)$ singularity at $z_{2}=0$, which by (5.9) will intersect the contracting locus at a $\mathbb{P}^{1}$. We can do this by choosing coefficients in (5.7) as follows:

$$
c_{23}=c_{33}=c_{43}=c_{44}=c_{64}=c_{65}=c_{68}=0
$$

keeping the rest of the $c_{i j}$ arbitrary.

\section{Mapping the landscape of singularities}

So far we have described how to analyze each embedding individually. In order to find actual examples, one needs to scan over a large class of toric ambient spaces, comparing their two dimensional faces to our desired target geometry. We will focus our efforts on the set of Calabi-Yau threefolds constructed by Kreuzer and Skarke [10], containing 473,800,776 reflexive polytopes. We refer to this set of Calabi-Yau manifolds as the KS landscape in what follows. Doing an exhaustive scan of such a big dataset requires the systematic use of computers; in section 6.7 we give a short overview of the computer tools we developed for performing this task.

The following set of questions, though by no means exhaustive, gives a flavor of how singular the landscape is. Summarizing the results, it is very simple to find singular CalabiYau spaces (including very singular Calabi-Yau spaces). Even relatively complicated Toric Lego models can be found in rather large numbers.

\subsection{How singular is the KS landscape?}

One of the most important questions one may try to answer concerns to what degree is the landscape singular. In our framework we attempt to answer this question by counting the number of interior points of the two dimensional faces of the $4 \mathrm{~d}$ polytopes. Locally, each of this interior points corresponds to a zero-size 4-cycle. Naturally, whether this 4-cycle has zero size or not depends on where we are in the Kähler cone of the Calabi-Yau hypersurface, and a priori it may be the case that we cannot contract the 4-cycle without sending the whole Calabi-Yau to zero size. Nevertheless, as we have seen in the examples in section 2, it seems to be possible to contract many four cycles even in relatively complex examples.

According to this philosophy, we scanned the KS landscape counting the number of internal points in each of the two-dimensional faces. The result is displayed in figure 9 .

One particularly remarkable feature of figure 9 is its smoothness. Despite the finite sample size, introducing some noise as we go towards low frequencies, it does definitely look like the singularity structure follows a well defined probability distribution. It would be interesting to see whether this structure persists as we consider other datasets (natural generalizations are higher dimensional reflexive polytopes). We leave this as an empirical observation for the moment.

The distribution of interior points peaks at 0 interior points, but it is rather common having just a few interior points. For reference, in table 2 we include the number of two dimensional faces with less than 10 interior points. 


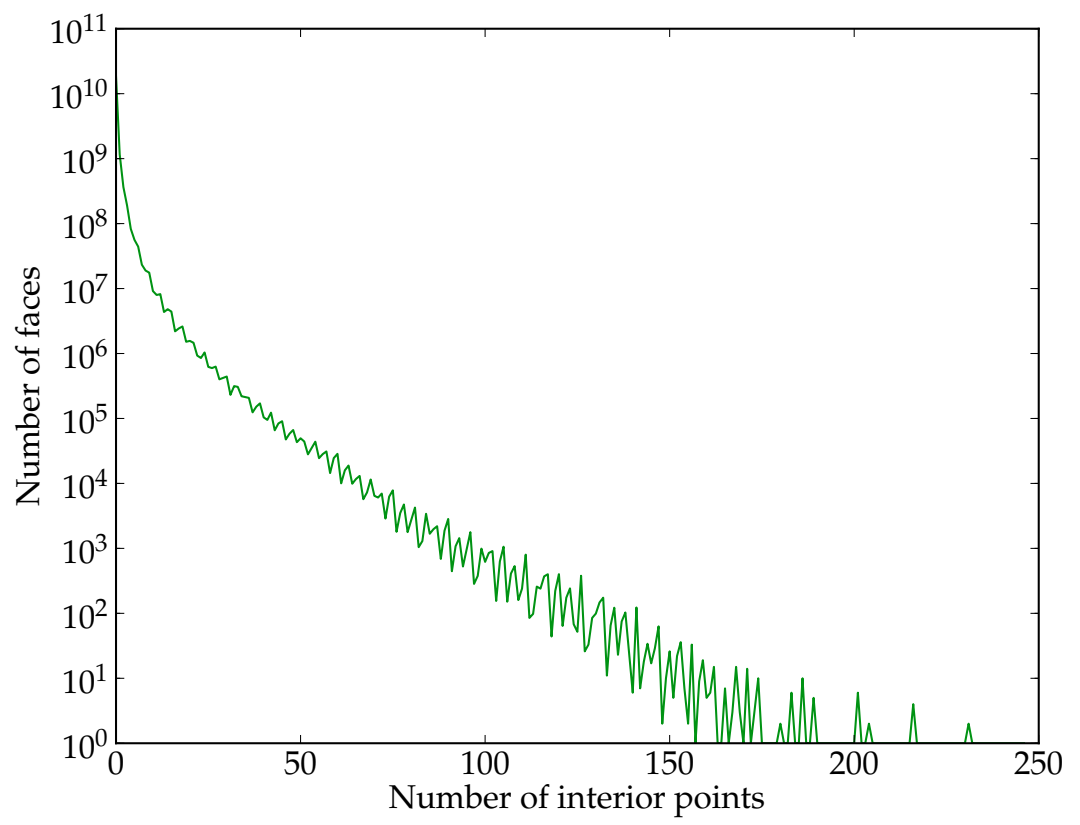

Figure 9. Number of two dimensional faces in the KS landscape having the given number of interior points.

\begin{tabular}{|c|c|}
\hline Interior points & Number of faces \\
\hline 0 & $18,348,252,546$ \\
1 & $1,160,340,121$ \\
2 & $364,176,255$ \\
3 & $188,901,035$ \\
4 & $82,981,171$ \\
5 & $56,180,491$ \\
6 & $44,224,288$ \\
7 & $23,299,165$ \\
8 & $18,939,629$ \\
9 & $17,560,669$ \\
\hline
\end{tabular}

Table 2. Number of two dimensional faces with less than 10 interior points.

\subsection{Singularities vs. Hodge numbers}

From the combinatorial formula [62] for the Hodge numbers of toric hypersurfaces

$$
\begin{aligned}
& h^{11}(X)=\#(\nabla)-4-1-\sum_{\operatorname{codim}(\nu)=1} \operatorname{Int}(\nu)+\sum_{\operatorname{codim}(\nu)=2} \operatorname{Int}(\nu) \operatorname{Int}\left(\nu^{*}\right) \\
& h^{21}(X)=\#(\Delta)-4-1-\sum_{\operatorname{codim}(\delta)=1} \operatorname{Int}(\delta)+\sum_{\operatorname{codim}(\delta)=2} \operatorname{Int}(\delta) \operatorname{Int}\left(\delta^{*}\right)
\end{aligned}
$$




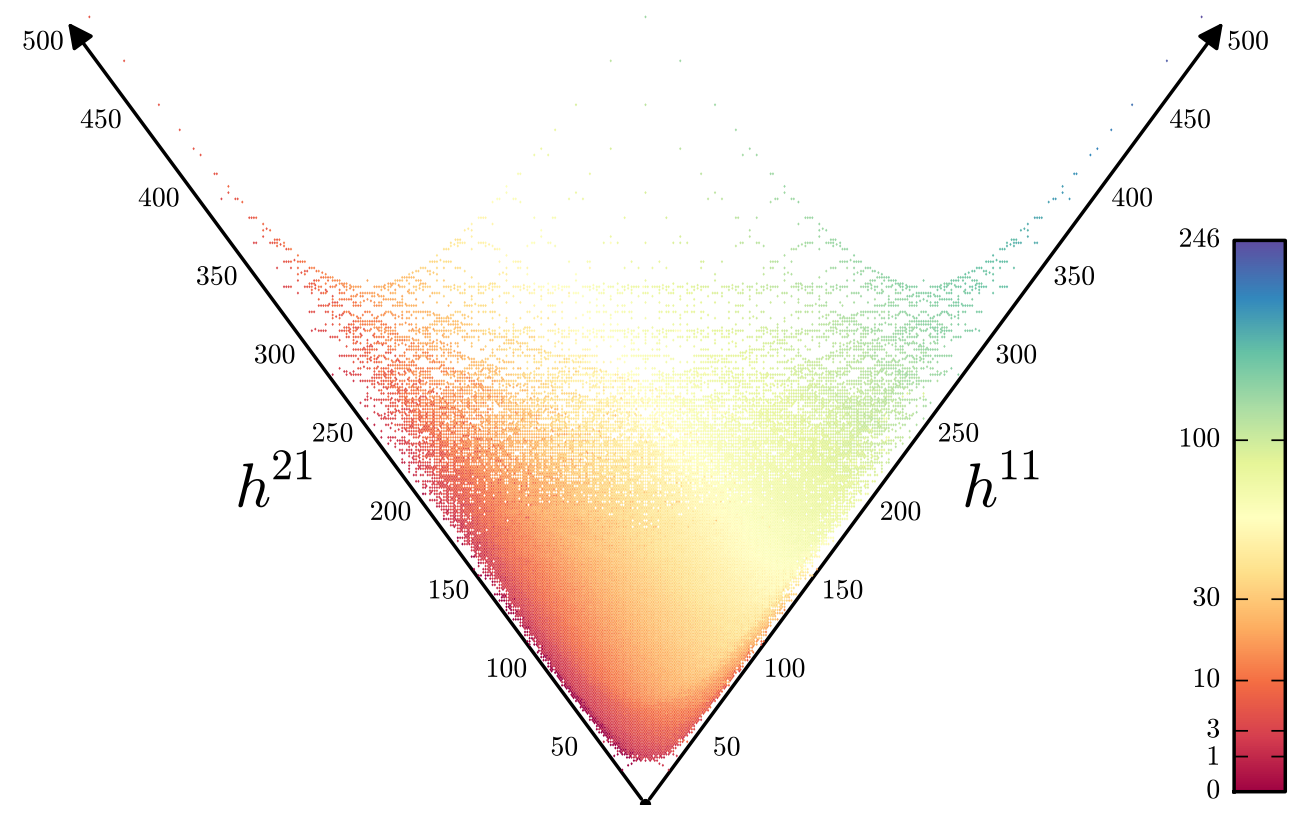

Figure 10. Maximal number of integral points on a single 2-face for each Hodge number pair.

it is clear that the number of internal points in various faces of the polytope is related to the Hodge numbers of the Calabi-Yau hypersurface. In figure 10, we plot the number of internal points by Hodge numbers. The plot shows no mirror symmetry. In fact, the number of internal points is mostly related to $h^{11}$ only.

\subsection{The most singular polytope}

A natural question is how singular can we make the space. This has been partially answered above, by studying the interior point structure of the KS landscape. In this section we focus explicitly on the most singular polytope that we found. Here we define most singular as the polytope containing the two-dimensional face with the largest number of interior points. From figure 10 we see that this is the rightmost manifold which has Hodge numbers $(491,11)$. This most singular polytope $\Sigma$ is defined by the following vertices:

\begin{tabular}{rrrrr}
$v_{1}$ & $v_{2}$ & $v_{3}$ & $v_{4}$ & $v_{5}$ \\
\hline 1 & -1 & 1 & 1 & 1 \\
1 & 1 & -2 & 1 & 1 \\
1 & 1 & 1 & -6 & 1 \\
1 & 1 & 1 & 1 & -83
\end{tabular}

In particular, its most singular two-dimensional face is defined by $v_{1}, v_{4}$ and $v_{5}$, and it has 246 integral points. Locally, it defines a $\mathbb{C}^{3} /\left(\mathbb{Z}_{84} \times \mathbb{Z}_{7}\right)$ singularity. In fact, all 10 of the two dimensional faces of this polytope are of the form $\mathbb{C}^{3} /\left(\mathbb{Z}_{p} \times \mathbb{Z}_{q}\right)$, with

$$
\{(p, q)\}=\{(84,3),(84,7),(7,3),(7,3),(84,2),(3,2),(3,2),(7,2),(7,2),(1,1)\}
$$




\begin{tabular}{|c|c|}
\hline Singularity & Number of faces \\
\hline$d P_{0}$ & $6,438,735$ \\
$d P_{1}$ & $33,073,205$ \\
$d P_{2}$ & $60,732,256$ \\
$d P_{3}$ & $17,085,648$ \\
\hline
\end{tabular}

Table 3. Local del Pezzo singularities.

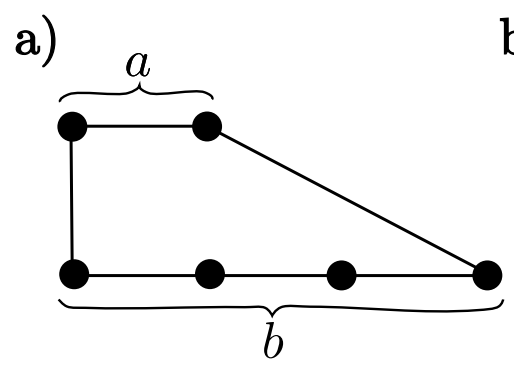

b)

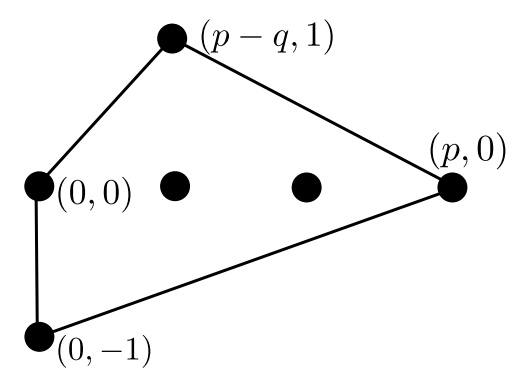

Figure 11. a) Toric diagram for a $L^{(a, b, a)}$ singularity. The particular example shown is $L^{(2,4,2)}$. b) Toric diagram for $Y^{(p, q)}$. We have shown $Y^{(3,2)}$.

Let us remark in passing that the Calabi-Yau manifold $X$ obtained from $\Sigma$ is both elliptically and K3 fibered, and thus gives an interesting background for studying heterotic/Ftheory duality. It was in fact studied in [63], where it was found that compactifying F-theory on $X$ gives rise to a 6 d theory with gauge group $G=E_{8}^{17} \times F_{4}^{16} \times G_{2}^{32} \times \mathrm{SU}(2)^{32}$ and $n_{T}=193 .^{15}$

\subsection{Toric del Pezzo singularities}

Calabi-Yau spaces with del-Pezzo singularities are of particular interest to model building $[4,6,11-15,18,42]$. The results of a scan for two dimensional faces being the toric diagrams of del Pezzo singularities is displayed in table 3.

Note that some care is required in interpreting the results in table 3 . While the results denote two-dimensional faces which correspond precisely to the toric diagrams for del Pezzo surfaces, there is a much larger number of faces that contain the del Pezzo diagrams. If one has one such face containing a del Pezzo singularity, a series of small resolutions of the singularity may leave behind precisely the desired del Pezzo. A similar caveat applies to the rest of the discussion in this section.

\section{5 $Y^{(p, q)}$ and $L^{(a, b, a)}$ cones}

The $L^{(a, b, a)}$ family of local toric singularities generalizes the conifold and the SPP singularities (these geometries belong to the general $L^{(a, b, c)}$ family [64, 65], which also comprises the $Y^{(p, q)}$ geometries below). The generic toric diagram for one such singularity is displayed in figure 11a, it has vertices at $(0,0),(0,1),(a, 1)$ and $(b, 0)$. We choose the convention $a \leq b$.

\footnotetext{
${ }^{15}$ We would like to thank W. Taylor for a remark about this point.
} 


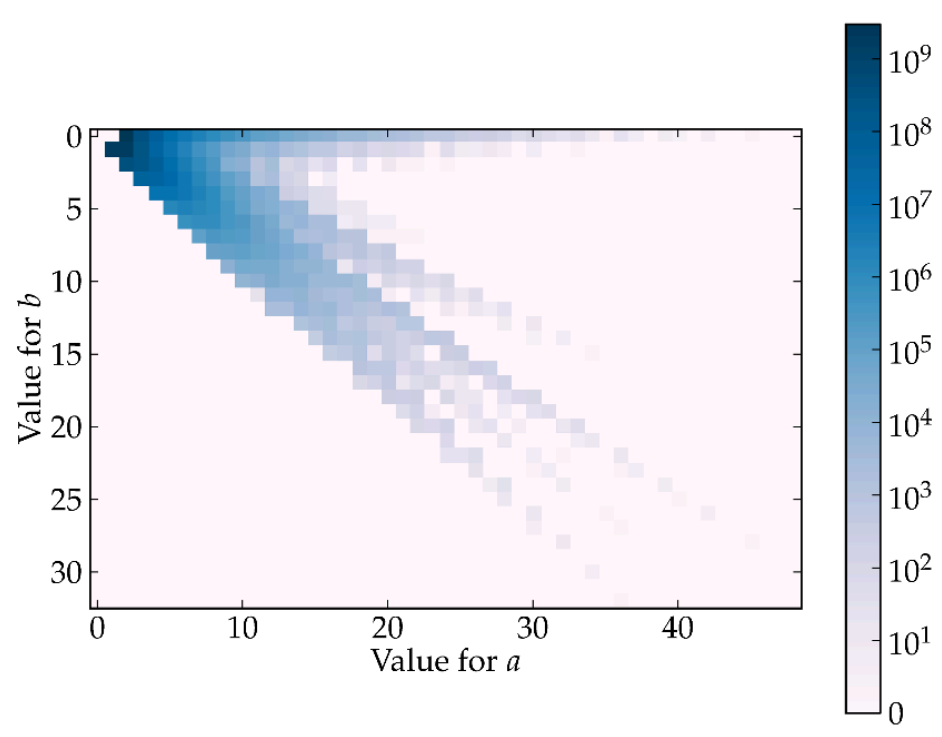

Figure 12. Plot of the number of local $L^{(a, b, a)}$ geometries in the KS landscape. $L^{(0,1,0)}$ is just a copy of flat space, so we have omitted it from the plot.

These singularities find a particularly nice model building use in the context of metastable supersymmetry breaking, see for example [36, 37]. We have performed an exhaustive scan over the KS landscape for these singularities, with the results shown in figure 12.

Notice that very singular $L^{(a, b, a)}$ singularities tend to have either $a \approx b$, i.e. they are roughly orbifolds of the conifold, or $b \approx 0$, in which case are geometries of the form $\mathbb{C}^{2} / \mathbb{Z}_{n} \times \mathbb{C}$.

A related large class of local singularities that we studied are the $Y^{(p, q)}$ cones $[66-$ 68]. They can also play an important role in the local model building approach (see for example [69] for applications to metastable supersymmetry breaking) and also in $\mathcal{N}=1$ AdS/CFT. We show the corresponding toric diagram in figure 11b. We have collected the results of the exhaustive scan for these singularities in table 4 .

\subsection{Toric Lego models}

Our original motivation in approaching the problem of finding global embeddings was to see whether there was any obstruction to the existence of global embeddings for Toric Lego singularities. As representative examples, we have performed exhaustive scans for a model consisting of three $d P_{0}$ sectors (given by the toric diagram in figure 8), and one three sector model $\left(d P_{0}+d P_{0}+d P_{1}\right)$ considered previously in [7], which we reproduce in figure 13 for convenience.

For the model in figure 8 we found 41,799 polytopes having that toric diagram as one of their two-dimensional faces, and 292,691 polytopes has figure 13 as one of their two dimensional faces. ${ }^{16}$

\footnotetext{
${ }^{16}$ We did not check how many of these polytopes respect the $\mathbb{Z}_{2}$ permutation involution described in section 4.3. As we describe in the conclusions, those polytopes not compatible with the involution could still work as embeddings in a more intrinsically F-theoretical approach.
} 


\begin{tabular}{|c|cccccccccccc|}
\hline & $q=0$ & 1 & 2 & 3 & 4 & 5 & 6 & 7 & 8 & 9 & 10 & 11 \\
\hline$Y^{2, q}$ & 12175355 & 33073205 & & & & & & & & & & \\
$Y^{3, q}$ & 203038 & 531568 & 311508 & & & & & & & & \\
$Y^{4, q}$ & 13123 & 14868 & 19772 & 10553 & & & & & & & \\
$Y^{5, q}$ & 200 & 672 & 357 & 632 & 351 & & & & & & \\
$Y^{6, q}$ & 114 & 129 & 171 & 130 & 167 & 129 & & & & & \\
$Y^{7, q}$ & 20 & 38 & 35 & 38 & 35 & 38 & 35 & & & & \\
$Y^{8, q}$ & 14 & 19 & 20 & 19 & 20 & 19 & 20 & 19 & & & \\
$Y^{9, q}$ & 3 & 5 & 5 & 5 & 5 & 5 & 5 & 5 & 5 & & & \\
$Y^{10, q}$ & 2 & 3 & 3 & 3 & 3 & 3 & 3 & 3 & 3 & 3 & & \\
$Y^{11, q}$ & 0 & 0 & 0 & 0 & 0 & 0 & 0 & 0 & 0 & 0 & 0 & \\
$Y^{12, q}$ & 1 & 1 & 1 & 1 & 1 & 1 & 1 & 1 & 1 & 1 & 1 & 1 \\
\hline
\end{tabular}

Table 4. Number of local $Y^{p, q}$ cones. No $Y^{p, q}$ spaces with $p>12$ were found.

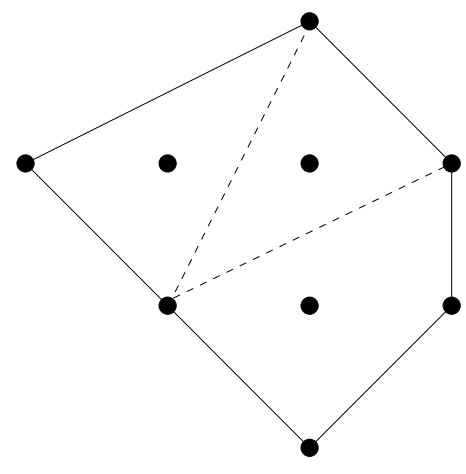

Figure 13. Three sector $\left(d P_{0}+d P_{0}+d P_{1}\right)$ model considered in [7].

\subsection{Computer implementation}

The scans performed above were done on a 2.5 GHZ Intel Core 2 Duo, with 4 GB of 667 $\mathrm{MHz}$ DDR2 SDRAM (MacBook Pro), using PALP [70] to generate the polytopes and some custom $\mathrm{C}$ code to analyze the polytopes for particular singularities. The code is available at the address http://cern.ch/inaki/scan.tar.gz together with the results of the scan. The whole scan was completed in 4 days, with the two cores running in parallel.

In order to analyze the resulting set of polytopes, we used Sage [71], and in particular its toric varieties package [72].

\section{Conclusions and generalizations}

In this paper we have provided a prescription for how to find global realizations of local models of gauge and matter content in which D-branes are placed at toric singularities. In particular, we focus on hypersurfaces in four dimensional toric varieties, using the complete classification of such a class of ambient spaces [10]. The global models are then either 
given in terms of type IIB orientifolds on compact Calabi-Yau manifolds or by considering a Calabi-Yau four-fold elliptically fibered over a compact three-dimensional base. Our construction provides a large class of models in which we can explore further important issues such as moduli stabilization in flux compactifications on type IIB orientifolds. In this way, our results link the rich literature on models at local toric singularities (see e.g. $[5,18,73,74])$ with that of the wealth of results on moduli stabilization (see $[35,75-$ 78] for some of the classical results, and [79-85] for some interesting recent developments). These models also present a way of embedding local singularities into the very large class of backgrounds appearing in F-theory. As opposed to the usual F-theory construction of GUTs, in our construction the MSSM sector comes from a very singular region, which cannot be described in F-theory. This, of course, does not preclude the bulk F-theory to have some interesting dynamics of its own.

In our discussion of the landscape, we found that singularities in the Calabi-Yau are generic. One can of course blow up to a smooth manifold, but since this breaks part of the symmetry once we have branes around, one expects that configurations with branes at singularities are dynamically preferred [86]. It is then an interesting and natural question to explore what happens in very singular manifolds. Our work provides some tools for analyzing this question in the context of toric geometries.

Finally, we have focused on the tools and the generic discussion. We hope to come back to the construction of realistic models using the techniques presented in this paper.

\subsection{More general F-theory bases}

While in this paper we have proceeded mostly along the traditional IIB route, it is clear that one can directly construct many bases of elliptically fibered fourfolds having the required structure for the construction in this paper to apply. Let us assume that we construct the basis $\mathcal{B}_{3}$ as a hypersurface in an ambient toric space $\mathcal{A}_{\nabla},{ }^{17}$ described by a polytope $\nabla$ (this setup has been recently analyzed in [87-89]). Then we require that:

1. $\nabla$ has a two-dimensional face describing the singularity that we want to embed.

2. The restriction of $K_{\mathcal{B}_{3}}$, the canonical class of $\mathcal{B}_{3}$, to the contracting locus is trivial.

The first condition follows from the same arguments as in section 2.1. The second condition is slightly more subtle, and encodes the fact that in the neighborhood of the singularity there are no orientifolds. This is the case in many of the examples in the literature. Condition 2 then ensures that close to the singularity the space is Calabi-Yau, as in section 5.1. This last condition can easily be modified if one wants local models with orientifolds. One just has to compute the canonical class of the base after the involution, similar to the computation that we did in section 4.3 .

\subsection{Complete intersections and toroidal orbifolds}

It would be interesting to extend our searches to the more general class of complete intersection Calabi-Yau manifolds (CICYs) in a toric variety. Unfortunately, the complete

\footnotetext{
${ }^{17}$ The extension to complete intersections proceeds very similarly to that in section 7.2 .
} 
classification of higher dimensional toric ambient spaces $(n>4$, with $n$ the complex dimension) is unknown, though partial results are known for $n=5$ and $n=6$ [90]. While this implies that it is at present not possible to carry out a complete search for the most general class of Calabi-Yau manifolds, our construction, and in particular the general discussion in section 2.1, carries over straightforwardly to the case of CICYs, mutatis mutandis.

Consider a $n$-dimensional reflexive polytope $\nabla$, together with some nef partition, giving a three-dimensional Calabi-Yau space $M_{X}$ as a complete intersection on $\mathcal{A}_{\nabla}$, the toric space associated to $\nabla$. Assume that we find that the toric diagram for the local toric CalabiYau $X$ in which we are interested appears as one of the two-dimensional faces of $\nabla$, or equivalently, the toric fan for $X$ is embedded as a three-dimensional cone in $\nabla$. Then $\mathcal{A}_{\nabla}$ has a local patch of the form $X \times\left(\mathbb{C}^{*}\right)^{n-3}$, and the set of hypersurfaces defining the complete intersection will generically give rise to a copy of $X$ embedded in $M_{X}$.

This observation allows one to study toroidal orbifolds using our methods. The only requirement is being able to describe the toroidal orbifold of interest as a complete intersection in an ambient toric variety. We refer the reader to the nice recent paper [91] for a careful analysis of how, and in which cases, it is possible to realize toroidal orbifolds as CICYs.

\section{Acknowledgments}

We would like to thank Mirjam Cvetič, Andrés Collinucci and Ángel Uranga for enlightening discussions. P.B. acknowledges the hospitality of the theory group at CERN, where this project was initiated and completed, as well as the Dublin Institute for Advanced Studies, the University of Pennsylvania, the KITPC, Beijing and the Berkeley Center for Theoretical Physics. V.B. acknowledges the hospitality of the KITP (which is supported in part by the National Science Foundation under Grant No. NSF PHY05-51164) during the final stage of this project. I.G.-E. acknowledges the hospitality of the HKUST Institute for Advanced Study and the University of New Hampshire, where parts of this work were completed. I.G.-E. would also like to thank N. Hasegawa for kind encouragement and support. The work of P.B. is supported by the NSF CAREER grant PHY-0645686 and by the University of New Hampshire through its Faculty Scholars Award Program.

Open Access. This article is distributed under the terms of the Creative Commons Attribution License which permits any use, distribution and reproduction in any medium, provided the original author(s) and source are credited.

\section{References}

[1] M.R. Douglas and G.W. Moore, D-branes, quivers and ALE instantons, hep-th/9603167 [INSPIRE].

[2] I.R. Klebanov and E. Witten, Superconformal field theory on three-branes at a Calabi-Yau singularity, Nucl. Phys. B 536 (1998) 199 [hep-th/9807080] [INSPIRE].

[3] D.R. Morrison and M.R. Plesser, Nonspherical horizons. 1., Adv. Theor. Math. Phys. 3 (1999) 1 [hep-th/9810201] [INSPIRE]. 
[4] G. Aldazabal, L.E. Ibáñez, F. Quevedo and A. Uranga, D-branes at singularities: A Bottom up approach to the string embedding of the standard model, JHEP 08 (2000) 002 [hep-th/0005067] [INSPIRE].

[5] D. Berenstein, V. Jejjala and R.G. Leigh, The Standard model on a D-brane, Phys. Rev. Lett. 88 (2002) 071602 [hep-ph/0105042] [INSPIRE].

[6] H. Verlinde and M. Wijnholt, Building the standard model on a D3-brane, JHEP 01 (2007) 106 [hep-th/0508089] [INSPIRE].

[7] V. Balasubramanian, P. Berglund and I. Garcia-Etxebarria, Toric Lego: A Method for modular model building, JHEP 01 (2010) 076 [arXiv:0910.3616] [INSPIRE].

[8] I. Garcia-Etxebarria, F. Saad and A.M. Uranga, Quiver gauge theories at resolved and deformed singularities using dimers, JHEP 06 (2006) 055 [hep-th/0603108] [INSPIRE].

[9] I. Garcia-Etxebarria, F. Saad and A.M. Uranga, Local models of gauge mediated supersymmetry breaking in string theory, JHEP 08 (2006) 069 [hep-th/0605166] [INSPIRE].

[10] M. Kreuzer and H. Skarke, Complete classification of reflexive polyhedra in four-dimensions, Adv. Theor. Math. Phys. 4 (2002) 1209 [hep-th/0002240] [INSPIRE].

[11] R. Donagi and M. Wijnholt, Model Building with F-theory, Adv. Theor. Math. Phys. 15 (2011) 1237 [arXiv: 0802.2969] [INSPIRE].

[12] C. Beasley, J.J. Heckman and C. Vafa, GUTs and Exceptional Branes in F-theory - I, JHEP 01 (2009) 058 [arXiv:0802.3391] [INSPIRE].

[13] H. Hayashi, R. Tatar, Y. Toda, T. Watari and M. Yamazaki, New Aspects of Heterotic-F Theory Duality, Nucl. Phys. B 806 (2009) 224 [arXiv:0805.1057] [INSPIRE].

[14] C. Beasley, J.J. Heckman and C. Vafa, GUTs and Exceptional Branes in F-theory - II: Experimental Predictions, JHEP 01 (2009) 059 [arXiv:0806.0102] [INSPIRE].

[15] R. Donagi and M. Wijnholt, Breaking GUT Groups in F-theory, arXiv:0808.2223 [INSPIRE].

[16] D.-E. Diaconescu, B. Florea, S. Kachru and P. Svrček, Gauge: mediated supersymmetry breaking in string compactifications, JHEP 02 (2006) 020 [hep-th/0512170] [INSPIRE].

[17] M. Buican, D. Malyshev, D.R. Morrison, H. Verlinde and M. Wijnholt, D-branes at Singularities, Compactification and Hypercharge, JHEP 01 (2007) 107 [hep-th/0610007] [INSPIRE].

[18] M.J. Dolan, S. Krippendorf and F. Quevedo, Towards a Systematic Construction of Realistic D-brane Models on a del Pezzo Singularity, JHEP 10 (2011) 024 [arXiv:1106.6039] [INSPIRE].

[19] R. Blumenhagen, M. Cvetič, P. Langacker and G. Shiu, Toward realistic intersecting D-brane models, Ann. Rev. Nucl. Part. Sci. 55 (2005) 71 [hep-th/0502005] [InSPIRE].

[20] J. Kumar and J.D. Wells, Surveying standard model flux vacua on $T^{6} / Z(2) \times Z(2)$, JHEP 09 (2005) 067 [hep-th/0506252] [INSPIRE].

[21] R. Blumenhagen, F. Gmeiner, G. Honecker, D. Lüst and T. Weigand, The Statistics of supersymmetric D-brane models, Nucl. Phys. B 713 (2005) 83 [hep-th/0411173] [INSPIRE].

[22] F. Gmeiner, R. Blumenhagen, G. Honecker, D. Lüst and T. Weigand, One in a billion: MSSM-like D-brane statistics, JHEP 01 (2006) 004 [hep-th/0510170] [INSPIRE]. 
[23] M.R. Douglas and W. Taylor, The Landscape of intersecting brane models, JHEP 01 (2007) 031 [hep-th/0606109] [INSPIRE].

[24] F. Gmeiner and G. Honecker, Mapping an Island in the Landscape, JHEP 09 (2007) 128 [arXiv: 0708.2285] [INSPIRE].

[25] F. Gmeiner and G. Honecker, Millions of Standard Models on $Z_{6}^{\prime}$ ?, JHEP 07 (2008) 052 [arXiv:0806.3039] [INSPIRE].

[26] W. Fulton, Introduction to toric varieties, Annals of mathematics studies, Princeton University Press, Princeton, NJ, U.S.A. (1993).

[27] K. Hori et al., Mirror symmetry, Clay Mathematics Monographs, CMI/AMS (2003).

[28] V. Bouchard, Lectures on complex geometry, Calabi-Yau manifolds and toric geometry, hep-th/0702063 [INSPIRE].

[29] D.A. Cox, J.B. Little and H.K. Schenck, Toric Varieties, Graduate Studies in Mathematics, AMS (2011)

[30] P. Candelas, A. Font, S.H. Katz and D.R. Morrison, Mirror symmetry for two parameter models. 2., Nucl. Phys. B 429 (1994) 626 [hep-th/9403187] [InSPIRE].

[31] D.R. Morrison and C. Vafa, Compactifications of F-theory on Calabi-Yau threefolds. 2., Nucl. Phys. B 476 (1996) 437 [hep-th/9603161] [inSPIRE].

[32] V. Braun, Toric Elliptic Fibrations and F-theory Compactifications, arXiv:1110.4883 [INSPIRE].

[33] E. Witten, Phases of $N=2$ theories in two-dimensions, Nucl. Phys. B 403 (1993) 159 [hep-th/9301042] [INSPIRE].

[34] P.S. Aspinwall, B.R. Greene and D.R. Morrison, Multiple mirror manifolds and topology change in string theory, Phys. Lett. B 303 (1993) 249 [hep-th/9301043] [INSPIRE].

[35] V. Balasubramanian, P. Berglund, J.P. Conlon and F. Quevedo, Systematics of moduli stabilisation in Calabi-Yau flux compactifications, JHEP 03 (2005) 007 [hep-th/0502058] [INSPIRE].

[36] R. Argurio, M. Bertolini, S. Franco and S. Kachru, Gauge/gravity duality and meta-stable dynamical supersymmetry breaking, JHEP 01 (2007) 083 [hep-th/0610212] [INSPIRE].

[37] R. Argurio, M. Bertolini, S. Franco and S. Kachru, Meta-stable vacua and D-branes at the conifold, JHEP 06 (2007) 017 [hep-th/0703236] [INSPIRE].

[38] D.-E. Diaconescu and J. Gomis, Fractional branes and boundary states in orbifold theories, JHEP 10 (2000) 001 [hep-th/9906242] [INSPIRE].

[39] M.R. Douglas, B. Fiol and C. Romelsberger, Stability and BPS branes, JHEP 09 (2005) 006 [hep-th/0002037] [INSPIRE].

[40] M.R. Douglas, B. Fiol and C. Romelsberger, The Spectrum of BPS branes on a noncompact Calabi-Yau, JHEP 09 (2005) 057 [hep-th/0003263] [INSPIRE].

[41] F. Cachazo, B. Fiol, K.A. Intriligator, S. Katz and C. Vafa, A Geometric unification of dualities, Nucl. Phys. B 628 (2002) 3 [hep-th/0110028] [InSPIRE].

[42] M. Wijnholt, Large volume perspective on branes at singularities, Adv. Theor. Math. Phys. 7 (2004) 1117 [hep-th/0212021] [INSPIRE].

[43] P.S. Aspinwall, D-branes on Calabi-Yau manifolds, hep-th/0403166 [INSPIRE]. 
[44] C.P. Herzog, Seiberg duality is an exceptional mutation, JHEP 08 (2004) 064 [hep-th/0405118] [INSPIRE].

[45] P.S. Aspinwall and I.V. Melnikov, D-branes on vanishing del Pezzo surfaces, JHEP 12 (2004) 042 [hep-th/0405134] [INSPIRE].

[46] C.P. Herzog and R.L. Karp, Exceptional collections and D-branes probing toric singularities, JHEP 02 (2006) 061 [hep-th/0507175] [INSPIRE].

[47] A. Hanany, C.P. Herzog and D. Vegh, Brane tilings and exceptional collections, JHEP 07 (2006) 001 [hep-th/0602041] [INSPIRE].

[48] D.S. Freed and E. Witten, Anomalies in string theory with D-branes, Asian J. Math 3 (1999) 819 [hep-th/9907189] [INSPIRE].

[49] S.H. Katz and E. Sharpe, D-branes, open string vertex operators and Ext groups, Adv. Theor. Math. Phys. 6 (2003) 979 [hep-th/0208104] [INSPIRE].

[50] T. Bridgeland, A. King and M. Reid, Mukai implies McKay: the McKay correspondence as an equivalence of derived categories, [math/9908027].

[51] A. Hanany and K.D. Kennaway, Dimer models and toric diagrams, hep-th/0503149 [INSPIRE].

[52] S. Franco, A. Hanany, K.D. Kennaway, D. Vegh and B. Wecht, Brane dimers and quiver gauge theories, JHEP 01 (2006) 096 [hep-th/0504110] [INSPIRE].

[53] S. Franco and D. Vegh, Moduli spaces of gauge theories from dimer models: Proof of the correspondence, JHEP 11 (2006) 054 [hep-th/0601063] [INSPIRE].

[54] A. Sen, F theory and orientifolds, Nucl. Phys. B 475 (1996) 562 [hep-th/9605150] [INSPIRE].

[55] A. Sen, F theory and the Gimon-Polchinski orientifold, Nucl. Phys. B 498 (1997) 135 [hep-th/9702061] [INSPIRE].

[56] A. Sen, Orientifold limit of F-theory vacua, Phys. Rev. D 55 (1997) 7345 [hep-th/9702165] [INSPIRE].

[57] A. Collinucci, New F-theory lifts, JHEP 08 (2009) 076 [arXiv:0812.0175] [INSPIRE].

[58] A. Collinucci, New F-theory lifts. II. Permutation orientifolds and enhanced singularities, JHEP 04 (2010) 076 [arXiv:0906.0003] [INSPIRE].

[59] R. Blumenhagen, T.W. Grimm, B. Jurke and T. Weigand, F-theory uplifts and GUTs, JHEP 09 (2009) 053 [arXiv:0906.0013] [INSPIRE].

[60] J. Tate, Algorithm for determining the type of a singular fiber in an elliptic pencil, in B. Birch and W. Kuyk eds., Modular Functions of One Variable IV, Lect. Notes Math. 476 (1975) 33.

[61] M. Bershadsky et al., Geometric singularities and enhanced gauge symmetries, Nucl. Phys. B 481 (1996) 215 [hep-th/9605200] [INSPIRE].

[62] V.V. Batyrev, Dual Polyhedra and Mirror Symmetry for Calabi-Yau Hypersurfaces in Toric Varieties, alg-geom/9310003.

[63] P. Candelas, E. Perevalov and G. Rajesh, Toric geometry and enhanced gauge symmetry of F-theory/heterotic vacua, Nucl. Phys. B 507 (1997) 445 [hep-th/9704097] [INSPIRE]. 
[64] M. Cvetič, H. Lü, D.N. Page and C. Pope, New Einstein-Sasaki spaces in five and higher dimensions, Phys. Rev. Lett. 95 (2005) 071101 [hep-th/0504225] [INSPIRE].

[65] D. Martelli and J. Sparks, Toric Sasaki-Einstein metrics on $S^{2} \times S^{3}$, Phys. Lett. B 621 (2005) 208 [hep-th/0505027] [INSPIRE].

[66] J.P. Gauntlett, D. Martelli, J. Sparks and D. Waldram, Supersymmetric AdS $S_{5}$ solutions of M-theory, Class. Quant. Grav. 21 (2004) 4335 [hep-th/0402153] [INSPIRE].

[67] J.P. Gauntlett, D. Martelli, J. Sparks and D. Waldram, Sasaki-Einstein metrics on $S^{2} \times S^{3}$, Adv. Theor. Math. Phys. 8 (2004) 711 [hep-th/0403002] [INSPIRE].

[68] D. Martelli and J. Sparks, Toric geometry, Sasaki-Einstein manifolds and a new infinite class of AdS/CFT duals, Commun. Math. Phys. 262 (2006) 51 [hep-th/0411238] [INSPIRE].

[69] I. Garcia-Etxebarria, F. Saad and A.M. Uranga, Supersymmetry breaking metastable vacua in runaway quiver gauge theories, JHEP 05 (2007) 047 [arXiv: 0704.0166] [INSPIRE].

[70] M. Kreuzer and H. Skarke, PALP: A Package for analyzing lattice polytopes with applications to toric geometry, Comput. Phys. Commun. 157 (2004) 87 [math/0204356] [INSPIRE].

[71] W. Stein et al., Sage Mathematics Software (Version 4.7), The Sage Development Team (2011) [http://www.sagemath.org].

[72] V. Braun and A. Novoseltsev, Toric Geometry in the Sage CAS, in preparation.

[73] G. Aldazabal, L.E. Ibáñez and F. Quevedo, $A D^{-}$brane alternative to the MSSM, JHEP 02 (2000) 015 [hep-ph/0001083] [INSPIRE].

[74] J. Cascales, M. Garcia del Moral, F. Quevedo and A. Uranga, Realistic D-brane models on warped throats: Fluxes, hierarchies and moduli stabilization, JHEP 02 (2004) 031 [hep-th/0312051] [INSPIRE].

[75] E. Witten, Nonperturbative superpotentials in string theory, Nucl. Phys. B 474 (1996) 343 [hep-th/9604030] [INSPIRE].

[76] S. Kachru, R. Kallosh, A.D. Linde and S.P. Trivedi, de Sitter vacua in string theory, Phys. Rev. D 68 (2003) 046005 [hep-th/0301240] [INSPIRE].

[77] R. Kallosh, A.-K. Kashani-Poor and A. Tomasiello, Counting fermionic zero modes on M5 with fluxes, JHEP 06 (2005) 069 [hep-th/0503138] [INSPIRE].

[78] E. Bergshoeff, R. Kallosh, A.-K. Kashani-Poor, D. Sorokin and A. Tomasiello, An Index for the Dirac operator on D3 branes with background fluxes, JHEP 10 (2005) 102 [hep-th/0507069] [INSPIRE].

[79] R. Blumenhagen, S. Moster and E. Plauschinn, Moduli Stabilisation versus Chirality for MSSM like Type IIB Orientifolds, JHEP 01 (2008) 058 [arXiv:0711.3389] [INSPIRE].

[80] A.M. Uranga, D-brane instantons and the effective field theory of flux compactifications, JHEP 01 (2009) 048 [arXiv:0808.2918] [INSPIRE].

[81] R. Blumenhagen, J. Conlon, S. Krippendorf, S. Moster and F. Quevedo, SUSY Breaking in Local String/F-Theory Models, JHEP 09 (2009) 007 [arXiv: 0906.3297] [INSPIRE].

[82] K. Bobkov, V. Braun, P. Kumar and S. Raby, Stabilizing All Kähler Moduli in Type IIB Orientifolds, JHEP 12 (2010) 056 [arXiv:1003.1982] [INSPIRE]. 
[83] T.W. Grimm, M. Kerstan, E. Palti and T. Weigand, On Fluxed Instantons and Moduli Stabilisation in IIB Orientifolds and F-theory, Phys. Rev. D 84 (2011) 066001 [arXiv:1105.3193] [INSPIRE].

[84] M. Cicoli, M. Kreuzer and C. Mayrhofer, Toric K3-Fibred Calabi-Yau Manifolds with del Pezzo Divisors for String Compactifications, JHEP 02 (2012) 002 [arXiv:1107.0383] [INSPIRE].

[85] M. Cicoli, C. Mayrhofer and R. Valandro, Moduli Stabilisation for Chiral Global Models, JHEP 02 (2012) 062 [arXiv:1110.3333] [INSPIRE].

[86] L. Kofman et al., Beauty is attractive: Moduli trapping at enhanced symmetry points, JHEP 05 (2004) 030 [hep-th/0403001] [INSPIRE].

[87] C.-M. Chen, J. Knapp, M. Kreuzer and C. Mayrhofer, Global SO(10) F-theory GUTs, JHEP 10 (2010) 057 [arXiv: 1005.5735] [InSPIRE].

[88] J. Knapp, M. Kreuzer, C. Mayrhofer and N.-O. Walliser, Toric Construction of Global F-theory GUTs, JHEP 03 (2011) 138 [arXiv:1101.4908] [INSPIRE].

[89] J. Knapp and M. Kreuzer, Toric Methods in F-theory Model Building, Adv. High Energy Phys. 2011 (2011) 513436 [arXiv: 1103.3358] [INSPIRE].

[90] M. Kreuzer, E. Riegler and D.A. Sahakyan, Toric complete intersections and weighted projective space, J. Geom. Phys. 46 (2003) 159 [math/0103214] [INSPIRE].

[91] M. Blaszczyk, S. Groot Nibbelink and F. Ruehle, Gauged Linear $\sigma$-models for toroidal orbifold resolutions, JHEP 05 (2012) 053 [arXiv:1111.5852] [INSPIRE]. 